\title{
HYPOCONVEXITY AND ESSENTIALLY $n$-NORMAL OPERATORS
}

BY

\author{
NORBERTO SALINAS
}

\begin{abstract}
In this paper a classifying structure for the class of essentially $n$-normal operators on a separable Hilbert space is introduced, and various invariance properties of this classifying structure are studied. The notion of a hypoconvex subset of the algebra $\mathfrak{T}_{n}$ of all complex $n \times n$ matrices is defined, and it is shown that the set of all equivalence classes of essentially $n$-normal operators (under a natural equivalence relation), whose reducing essential $n \times n$ matricial spectrum is a given hypoconvex set, forms an abelian group. It is also shown that this correspondence between hypoconvex subsets of $\mathfrak{T}_{n}$ and abelian groups is a homotopy invariant, covariant functor. This result is then used to prove that Toeplitz operators (on strongly pseudoconvex domains) which have homotopic continuous matricial symbols, are unitarily equivalent up to compact perturbation.
\end{abstract}

1. Introduction. This paper contains the detailed account of some results already announced in [30] and some further progress that we have made since the publication of that announcement. Our interest in the subject arose from the celebrated paper [10], and in [29] we started a program leading to generalizations of the various important concepts and fruitful ideas contained in [10]. In [26], [27] and [30] we continued our program with a more concrete purpose (see also [13]): to try to obtain a complete set of invariants for the class of essentially $n$-normal operators on separable Hilbert space up to a reasonable equivalence relation. The present paper might be regarded as a further step toward this goal.

Let $\mathcal{H}$ be a separable, infinite dimensional, complex Hilbert space and let $\mathcal{L}(\mathcal{F})$ denote the algebra of all (bounded, linear) operators on $\mathcal{H}$. Let $\mathcal{K}(\mathcal{F})$ denote the (closed) ideal of all compact operators on $\mathcal{H}$, and let $\pi_{\mathscr{S}}$ : $\mathcal{L}(\mathcal{F C}) \rightarrow 2(\mathcal{T C})$ denote the canonical homomorphism onto the quotient (Calkin) algebra $2(\mathcal{F})=\mathfrak{L}(\mathcal{H}) / \mathscr{K}(\mathcal{H})$ (when no confusion arises we will drop the subindex $\mathcal{T}$ of $\pi$ ).

Received by the editors October 3, 1977 and, in revised form, April 18, 1978 and July 5, 1978. AMS (MOS) subject classifications (1970). Primary 47B99, 46L05; Secondary 46M15, 47B35, $47 \mathrm{ClO}$.

Key words and phrases. Unitary equivalence up to compact perturbation, the reducing essential $n \times n$ matricial spectrum, extensions of $C^{*}$-algebras, the classification problem for essentially $n$-normal operators, Toeplitz operators on strongly pseudoconvex domains. 
Definition 1.1. Two operators $T$ and $S$ in $\mathcal{E}(\mathcal{H C})$ are said to be strongly equivalent, and we write $T \sim_{s} S$, if there exists a unitary operator $U$ in $\mathcal{L}(\mathcal{F})$ such that $U^{*} T U-S \in \mathcal{K}(\mathcal{F})$. On the other hand, $T$ and $S$ are said to be weakly equivalent, and we write $T \sim S$, if there exists $W$ in $\mathcal{L}(\mathcal{F C})$, such that $\pi W$ is unitary in $2(\mathcal{H})$ and $W^{*} T W-S \in \mathcal{K}(\mathcal{H C})$ (see [28]).

These natural equivalence relations on $\mathcal{E}(\mathcal{F C})$ were first introduced in [10, Corollary 4.4] where it was proved that for the class of operators with compact self-commutators (i.e. essentially normal operators) these two notions coincide. Furthermore, in [10, Theorem 4.3] it was shown that if two countable families of essentially commuting essentially normal operators are simultaneously weakly equivalent (that is with the same essentially unitary operator), then they are simultaneously strongly equivalent (i.e. with the same unitary operator). An extension of this result will be given in Lemma 3.3.

DEFINITION 1.2. An operator $T$ in $\mathcal{E}(\mathcal{H})$ will be called $n$-normal if there exists a unitary transformation $U: \mathcal{H} \rightarrow \mathcal{H} \otimes C^{n}$ such that the operator $U T U^{*}$ in $\mathcal{E}\left(\mathcal{H} \otimes C^{n}\right)\left(=\mathscr{E}(\mathcal{H}) \otimes \Re_{n}\right)$ is an $n \times n$ operator matrix with commuting normal entries. Hereafter, $\mathbf{C}^{n}$ denotes the standard $n$-dimensional complex vector space and $\mathfrak{N}_{n}$ denotes the algebra of all complex $n \times n$ matrices (which will be identified with $\mathcal{E}\left(C^{n}\right)$ ). An operator $T$ in $\mathcal{E}(\mathcal{F})$ will be called essentially $n$-normal if there exists a unitary transformation $U: \mathcal{C} \rightarrow$ $\mathcal{H} \otimes \mathrm{C}^{n}$ such that $\pi_{\mathcal{K} \otimes \mathrm{C}^{*}}\left(U T U^{*}\right)$ is an $n \times n$ matrix with commuting normal entries in $2(\mathcal{H})$.

REMARK 1.3. As we shall see in $\$ 4$, essentially $n$-normal operators arise naturally in the theory of generalized Toeplitz operators on strongly pseudoconvex domains. In [28] it was shown that two essentially binormal operators in $\mathcal{L}(\mathcal{H C})$ may be weakly equivalent but not strongly equivalent. Although the class of essentially $n$-normal operators is clearly left invariant by strong equivalence, the fact that weak equivalence also preserves essential $n$-normality is not so obvious and will be proved at the end of the present section.

In [27] it was shown that the reducing essential $n \times n$ spectrum constitutes a complete set of invariants, up to strong equivalence, for the class of $n$-normal operators in $\mathcal{L}(\mathcal{H C})$.

Definition 1.4. Let $T \in \mathcal{E}(\mathcal{H})$ and let $C^{*}(T)$ denote the $C^{*}$-algebra generated by $T$ and $1_{\mathscr{T}}$. The reducing essential $n \times n$ spectrum of $T$ is defined as $R_{e}^{n}(T)=\left\{L \in \Re_{n}\right.$ : there exists a unital *-homomorphism $\varphi: \pi\left[C^{*}(T)\right] \rightarrow$ $\mathscr{T}_{n}$ such that $\left.\varphi \pi T=L\right\}$ (see [26] and [13]).

REMARK 1.5. If $T$ is an essentially normal operator in $\mathcal{L}(\mathcal{H})$, then it follows from [29, \$6] that $R_{e}^{1}(T)$ coincides with the essential spectrum of $T$. Also, it is easy to show that if $T$ and $S$ are weakly equivalent operators, then $R_{e}^{n}(T)=$ $R_{e}^{n}(S)$. Notice that, in general, $R_{e}^{n}(T)=\varnothing$ for an arbitrary $T$ in $\mathcal{L}(\mathcal{H})$ (see [26, Remark 3.11]), but if $T$ is essentially $n$-normal, then necessarily $R_{e}^{n}(T) \neq$ $\varnothing$ [27, Theorem 3.5]. Thus, the reducing essential $n \times n$ matricial spectrum 
constitutes a nontrivial invariant, up to weak or strong equivalence, for the class of essentially $n$-normal operators in $\mathcal{L}(\mathcal{H C})$. Furthermore, it was shown in [27, Theorem 4.4] that if $T$ is an essentially $n$-normal operator in $\mathcal{L}(\mathcal{H})$, and $S$ is an $n$-normal operator on $\mathcal{H}$ such that $R_{e}^{n}(S) \subset R_{e}^{n}(T)$, then $T \oplus S \sim_{s} T$. In particular, two $n$-normal operators $T$ and $S$ are strongly equivalent if and only if $R_{e}^{n}(T)=R_{e}^{n}(S)$.

If $T$ is an arbitrary operator in $\mathcal{E}(\mathcal{F})$ such that $R_{e}^{n}(T)=X \neq \varnothing$, then the following properties are easy to verify:

(*) $X$ is a compact subset of $\mathscr{K}_{n}$ which is invariant under unitary conjugation in $\Re_{n}$.

(**) If $L_{j} \in X, 1<j \leqslant m$, and $P_{j}$ is a projection in $\pi_{n}$ (selfadjoint idempotent) such that $P_{j} L_{j}=L_{j} P_{j}, j=1,2, \ldots, m$, and $\sum_{j=1}^{m} P_{j}=1$, then $\sum_{j=1}^{m} P_{j} L_{j} \in X, m=1,2, \ldots$

DEFINITION 1.6. A nonempty subset $X$ of $\mathfrak{T}_{n}$ which satisfies conditions (*) and (**) will be called hypoconvex (see [30]). If $Y$ is an arbitrary nonempty compact subset of $\Re_{n}$, we denote by $\hat{Y}$ the smallest hypoconvex set containing $Y$ and we call it the hypoconvex hull of $Y$.

LEMMA 1.7. Let $T$ and $S$ be two essentially n-normal operators in $\mathcal{L}(\mathcal{H})$. Then, $R_{e}^{n}(T \oplus S)=\left[R_{e}^{n}(T) \cup R_{e}^{n}(S)\right]^{\text {. }}$.

Proof. Since $R_{e}^{n}(T \oplus S)$ satisfies conditions (*) and (**), one inclusion is obvious. For the other inclusion, let $L \in R_{e}^{n}(T \oplus S)$ and suppose that $L$ is written on some orthonormal basis of $C^{n}$ as the direct sum of its irreducible direct summands $L=\sum_{j=1}^{m} \oplus L_{j}$, where $L_{j} \in \mathfrak{N}_{n j}, \sum_{j=1}^{m} n_{j}=n$. Then, for each $j=1, \ldots, m$, there exists a unital $*$-homomorphism $\varphi_{j}: \pi C^{*}(T \oplus \mathrm{S}) \rightarrow$ $\Re_{n}$, such that $\varphi_{j} \pi(T \oplus S)=L_{j}$. Since $\varphi_{j}$ is an irreducible representation of $C^{*}[\pi(T \oplus S)]$ on $C^{3}$, from [17, Theorem 2.10.2] $\varphi_{j}$ can be dilated to an irreducible representation $\psi_{j}: C^{*}(T) \oplus C^{*}(S) \rightarrow \mathcal{L}\left(\mathcal{F C}_{j}^{\prime}\right)$, for some separable Hilbert space $\mathcal{H}_{j}^{\prime}, \mathrm{C}^{n_{j}} \subset \mathcal{F}_{j}^{\prime}$, such that $\mathrm{C}_{j}^{n}$ reduces $\psi_{j} A$, and $\psi_{j} A \mid \mathrm{C}^{\eta_{j}}=\varphi_{j} A$, for every $A \in C^{*}[\pi(T \oplus S)]$. Since $1 \oplus 0 \in C^{*}[\pi(T)] \oplus C^{*}[\pi(S)]$ and $\psi_{j}$ is irreducible, we deduce that either $1 \oplus 0$ or $0 \oplus 1$ belongs to ker $\psi_{j}$. Suppose, for instance, that $0 \oplus 1 \in \operatorname{ker} \psi_{j}$, and let $\theta_{j}$ be the restriction of $\psi_{j}$ to $C^{*}[\pi(T)]$ (via $1 \oplus 0)$. Then $L_{j}=\varphi_{j} \pi T=\left(\theta_{j} \pi T\right) \mid C^{\eta_{j}} \in R_{e}^{n}(T)$, and hence $R_{e}^{n-n_{3}}(T) \neq \varnothing$ [12]. It follows that $\sum_{j=1}^{m} \bigoplus L_{j} \in\left[R_{e}^{n}(T) \cup R_{e}^{n}(S)\right]^{n}$.

DefinItion 1.8. Let $X \subset \mathfrak{R}_{n}$ be a hypoconvex set and let $E N_{n}^{s}(X)$ [resp., $E N_{n}(X)$ ] be the set of all strong [resp., weak] equivalence classes [T] [resp., [T]] of essentially $n$-normal operators $T$ in $\mathcal{L}(\mathcal{H})$ such that $R_{e}^{n}(T)=X$.

REMARK 1.9. Given two essentially $n$-normal operators $T$ and $S$, it readily follows that $T \oplus S$ is also essentially $n$-normal. Thus, in view of Lemma 1.7, the following abelian operations can be defined on $E N_{n}^{s}(X)$ and $E N_{n}(X)$ : $[T]_{s}+[S]_{s}=[T \oplus S]_{s}$ and $[T]+[S]=[T \oplus S]$, where $T \oplus S$ has been identified with an operator in $E(F)$. From the results stated in Remark 1.5, it 
follows that $E N_{n}^{s}(X)$ and $E N_{n}(X)$ are abelian semigroups whose neutral elements consist of the strong equivalence class and weak equivalence class containing all $n$-normal operators $S$ such that $R_{e}^{n}(S)=X$. It is immediate that the correspondence $[T]_{s} \rightarrow[T]$ defines a surjective homomorphism $\Theta$ : $E N_{n}^{s}(X) \rightarrow E N_{n}(X)$.

The classification problem for essentially $n$-normal operators, up to weak equivalence [resp., strong equivalence], consists of computing $E N_{n}(X)$ [resp., $\left.E N_{n}^{s}(X)\right]$ for every hypoconvex set $X$. Although we do not solve the general problem in this paper, we present several interesting partial results, explicitly solving the classification problem for certain hypoconvex subsets of $\Re_{n}$. In $\$ 3$ we shall prove that both $E N_{n}^{s}(X)$ and $E N_{n}(X)$ are actually groups, and that $\operatorname{ker} \Theta \subset \mathrm{Z} / n \mathrm{Z}$. Furthermore, we shall show that $E N_{n}^{s}(\cdot)$ and $E N_{n}(\cdot)$ are homotopy invariant covariant functors from the category of all hypoconvex subsets of $\mathfrak{R}_{n}$ into the category of abelian groups. An interesting corollary of this result is that $E N_{n}^{s}\left(\mathscr{B}_{n}\right)=E N_{n}\left(\mathscr{B}_{n}\right)=\{0\}$ where $\mathscr{B}_{n}$ is the closed unit ball in $\Re_{n}$. In $\$ 4$ we use the homotopy invariance of $E N_{n}^{s}(X)$ to show that if $\varphi, \psi: \bar{\Omega} \rightarrow \Re_{n}$ are two continuous functions on a strongly pseudoconvex domain $\Omega$ (with smooth boundary) such that [range $\varphi \mid \partial \Omega]^{\wedge}=[\text { range } \psi \mid \partial \Omega]^{\wedge}=$

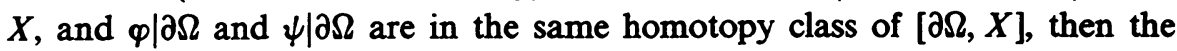
corresponding Toeplitz operators defined by $\varphi$ and $\psi$, respectively, are strongly equivalent (that is they are unitarily equivalent up to compact perturbation).

Our next task is to present a direct proof that $E N_{n}^{s}(X)$ and $E N_{n}(X)$ are not empty for every hypoconvex $X \subset \mathfrak{K}_{n}$. (An alternative proof, using extension theory, will be given in §3.) We shall need the following lemma whose proof (which we omit) can be obtained by using arguments similar to those employed in the proof of [26, Theorem 1.1].

LEMMA 1.10. Let $\mathscr{B} \subset \mathcal{L}(\mathcal{H})$ be a unital separable $C^{*}$-algebra and suppose there is a family $\left\{P_{k}: 1 \leqslant k \leqslant m\right\}$ with $m \leqslant x_{0}$, of mutually orthogonal projections in $E(\mathcal{H C})$ such that $\sum_{k=1}^{m} P_{k}=1_{\mathcal{X}}$ and $P_{k} B=B P_{k}$, for every $B \in$ $\mathscr{B}, 1<k<m$. If $\varphi: \mathscr{B} \rightarrow \Re_{n}$ is an irreducible representation of $\mathscr{B}$ on $\mathbf{C}^{n}$ such that $\mathscr{B} \cap \mathcal{H}(\mathcal{C}) \subset \operatorname{ker} \varphi$, then there exist $n^{2}$ partial isometries $W_{i, j}$ in $\mathcal{L}(\mathcal{H C}), 1<i, j<n$, which form a system of matrix units in $\mathcal{L}(\mathcal{I C})$ and satisfy:

(i) The projection $Q=\sum_{i=1}^{n} W_{i, i}$ has infinite rank and nullity,

(ii) $B Q-Q B \in \mathcal{K}(\mathcal{H})$, for every $B \in \mathscr{B}$,

(iii) $Q B Q-\sum_{i, j=1}^{n}[\varphi B]_{i, j} W_{i, j} \in \mathcal{K}(\mathcal{H C})$, where $[\varphi B]_{i, j}$ denotes the $(i, j)$ th entry of the matrix $\varphi B$, for every $B \in \mathscr{B}$,

(iv) $W_{i, j} P_{k}=P_{k} W_{i, j}$, for $1 \leqslant i, j<n, 1 \leqslant k \leqslant m$.

The following theorem generalizes a theorem of Weyl [35]. 
THEOREM 1.11. Let $\left\{L_{j}\right\}$ be a bounded sequence in $\mathfrak{T}_{n}$ and let $S$ be the (diagonal) n-normal operator defined by $S=\sum_{j=1}^{\infty} \oplus L_{j}$. Then, $R_{e}^{n}(S)$ coincides with the hypoconvex hull of the set of limit points of $\left\{L_{j}\right\}$.

Proof. If $L$ is a limit point of $\left\{L_{j}\right\}$, then it readily follows from [26, Corollary 3.7] (see also [13, Corollary 4.9]) that $L \in R_{e}^{n}(S)$, and since $R_{e}^{n}(S)$ is hypoconvex one inclusion follows. In order to prove the opposite inclusion it suffices to show that if $M$ is an irreducible matrix in $R_{e}^{m}(S)$, with $1<m \leqslant n$, then there exist a sequence of isometries $V_{j}: \mathbf{C}^{m} \rightarrow \mathbf{C}^{n}, j=$ $1,2, \ldots$, and a subsequence $\left\{L_{k_{j}}\right\}$ of $\left\{L_{k}\right\}$ such that $\lim _{j \rightarrow \infty} \| M-$ $V_{j}^{*} L_{k_{j}} V_{j}\|+\| L_{k_{j}} V_{j} V_{j}^{*}-V_{j} V_{j}^{*} L_{k_{j}} \|=0$. Let $P_{k}$ be the projection from $C^{n} \otimes l_{2}$ $=\mathbf{C}^{n} \oplus \mathbf{C}^{n} \oplus \ldots$ onto the $k$ th copy of $\mathbf{C}^{n}, k=1,2, \ldots$ Since $\left\{P_{k}\right\}$ is an orthogonal family of projections on $\mathbf{C}^{n} \otimes l_{2}$ such that $\sum_{k=1}^{\infty} P_{k}=1, P_{k} S=$ $S P_{k}$, for every $k=1,2, \ldots$, we can apply Lemma 1.10 to the $C^{*}$-algebra $C^{*}(S)$ and to the irreducible $m$-dimensional representation $\varphi: C^{*}(S) \rightarrow \Re_{n}$ determined by $\varphi S=M$. Therefore, it follows that there exist $m^{2}$ partial isometries $W_{i, j}$ on $\mathbf{C}^{n} \otimes l_{2}, 1 \leqslant i, j \leqslant m$, satisfying the corresponding versions of conditions (i)-(iv) of Lemma 1.10. Let $\left\{P_{k_{j}}\right\}$ be a subsequence of $\left\{P_{k}\right\}$ such that $P_{k_{j}} W_{1,1} \neq 0$ and let $e_{j}$ be a unit vector in range $W_{1,1} P_{k_{i}}, j=1,2, \ldots$ Also, let $f_{1}, \ldots, f_{m}$ be the standard bases for $\mathbf{C}^{m}$ and let $W_{j}: \mathbf{C}^{m} \rightarrow \mathbf{C}^{n} \otimes l_{2}$ be the linear transformation defined by $W_{j} f_{i}=W_{i, 1} e_{j}, 1<i<m, j=1$, $2, \ldots$ Since for each $j=1,2, \ldots$ the vectors $W_{1,1} e_{j}, W_{2,1} e_{j}, \ldots, W_{m, 1} e_{j}$ are orthonormal, it follows that $W_{j}$ is an isometry such that range $W_{j} \subset$ range $P_{k_{j}}$. Just as in the proof of [26, Theorem 3.2] we deduce that $\lim _{j \rightarrow \infty}\left(\left\|S W_{j}-W_{j} M\right\|+\left\|S^{*} W_{j}-W_{j} M^{*}\right\|\right)=0$. Now we let $V_{j}^{*}=$ $W_{j}^{*} \mid$ range $P_{k_{j}}, j=1,2, \ldots$ Then it follows that $\lim _{i \rightarrow \infty}\left(\left\|L_{k_{j}} V_{j}-V_{j} M\right\|+\right.$ $\left.\left\|L_{k_{j}}^{*} V_{j}-V_{j} M^{*}\right\|\right)=0$. This completes the proof of the theorem.

COROLlaRY 1.12. Let $X \subset \mathfrak{K}_{n}$ be hypoconvex. Then there exists an n-normal operator $S$ such that $R_{e}^{n}(S)=X$, and hence $E N_{n}(X)$ and $E N_{n}^{s}(X)$ are not empty.

Proof. Let $\left\{L_{j}\right\}$ be a sequence dense in $X$ whose terms are repeated infinitely often, and let $s=\sum_{j=1}^{\infty} \oplus L_{j}$. Then the corollary is a direct consequence of Theorem 1.11.

We close this section by showing a concrete form that weak equivalence takes on the class of essentially $n$-normal operators.

THEOREM 1.13. Let $T$ and $S$ be two operators on $\mathcal{H}$ such that $R_{e}^{n}(T) \neq \varnothing$ and $R_{e}^{n}(S) \neq \varnothing$. Then $T \sim S$ if and only if there exists an isometry $V$ in $\mathcal{L}(\mathcal{F C})$ with deficiency less than $n$ such that $V^{*} T V-S \in \mathcal{K}(\mathcal{F})$. 
PrOOF. Since isometries with finite deficiencies are essentially unitary (i.e. unitary in the Calkin algebra) one half of the theorem is obvious. In order to prove the other half suppose that there exists $W$ in $\mathcal{L}(\mathcal{H})$ with $\pi W$ unitary, such that $W^{*} T W-S \in \mathscr{K}(\mathcal{H})$. From [14] we can assume that $W$ is a partial isometry with finite index $k$. By perturbing $W$ with a finite rank partial isometry, we can further assume that $\min \left(\operatorname{dim} \operatorname{ker} W, \operatorname{dim} \operatorname{ker} W^{*}\right)=0$, i.e. that $W$ is an isometry or a coisometry. We suppose now that $W$ is a coisometry (the proof of the case in which $W$ is an isometry is similar and will be omitted). Let $j$ be a positive integer so that $0<n j-k<n$. Notice that $W^{*} T W$ is unitarily equivalent to an operator that can be expressed on [ker $\left.W^{*}\right] \oplus\left[\operatorname{ker} W^{*}\right]^{\perp}$ as $0 \oplus T$. Let $L_{1}, \ldots, L_{j}$ be in $R_{e}^{n}(T)$. It follows that there exists an isometry $V_{0}$ on $\left[C^{n} \otimes C^{j}\right] \oplus \mathcal{H}$, whose range is $\{0\} \oplus \mathcal{H}$, such that $V_{0}^{*}\left[\left(\Sigma_{i=1}^{j} \oplus L_{i}\right) \oplus T\right] V_{0}$ is unitarily equivalent to a compact perturbation of $S$. Since $\left(\Sigma_{i=1}^{j} \oplus L_{i}\right) \oplus T \sim_{s} T$ [27, Theorem 4.4], it readily follows that there exists an isometry $V$ in $\mathcal{L}(\mathcal{H})$ such that $V^{*} T V-S \in \mathcal{K}(\mathcal{H})$.

THEOREM 1.14. Let $T$ and $S$ be in $\mathcal{L}(\mathcal{T C})$ such that $S$ is an essentially n-normal operator and $T$ is weakly equivalent to $S$. Then, $T$ is also an essentially n-normal operator.

Proof. From Theorem 1.13 there exists an isometry $V$ in $\mathcal{E}(\mathcal{H})$ of deficiency less than $n$ such that $V^{*} T V-S \in \mathcal{K}(\mathcal{F})$. Thus, $T$ is unitarily equivalent to an operator of the form $0_{\mathscr{T}} \oplus\left(S_{i j}\right)$, where $\Re$ is a subspace of $\mathcal{C}$ of dimension less than $n$ and $\left(S_{i j}\right)$ is an $n \times n$ operator matrix acting on $\Re^{\perp}=\Re \otimes C^{n}$, with essentially commuting, essentially normal entries in $\mathcal{L}(\Re)$. Let $\mathcal{K}^{\prime}=\mathscr{N} \oplus \mathscr{T} \subset \mathcal{K}$ and let $W \in \mathcal{L}\left(\mathcal{H}^{\prime}\right)$ be any isometry such that range $W=\Re$. We define the unitary transformation $U: \Re \rightarrow \mathcal{H}^{\prime}$ $(=\Re \oplus \Re)$ by $U=W^{*} \mid \Re$. We then have $U^{*} W^{*} \mid \Re=1_{\mathscr{V}}$ and $W U$ : $\Re \rightarrow \mathcal{K}^{\prime}$ is the natural inclusion. Let $F: \Re \otimes \mathbf{C}^{n} \rightarrow \mathcal{K}^{\prime} \oplus\left(\Re \otimes \mathbf{C}^{n-1}\right)(=$ $\left.\Re \oplus\left(\Re \otimes C^{n}\right)=\mathscr{C}\right)$ be the isometry given by $F=W U \oplus\left(1_{\mathscr{X}} \otimes 1_{n-1}\right)$ where $1_{n-1}$ is the identity matrix in $\mathfrak{K}_{n-1}$. Then, codim range $F=$ codim range $W=\operatorname{dim} \Re<n$. We claim that $F\left(S_{i j}\right) F^{*}=0_{\Re} \oplus\left(S_{i j}\right)$. Indeed, given $x \in \Re, y \in \Re$ and $z \in \Re \otimes C^{n-1}$ we have

$$
\begin{aligned}
F\left(S_{i j}\right) F^{*}( & x \oplus y \oplus z) \\
& =\left(W U \oplus\left(1_{\Re} \otimes 1_{n-1}\right)\right)\left(S_{i j}\right)\left(W U \oplus\left(1_{\Re} \otimes 1_{n-1}\right)\right)^{*}(x \oplus y \oplus z) \\
& =\left(W U \oplus 1_{\Re} \otimes 1_{n-1}\right)\left[\left(S_{i j}\right)(y \oplus z)\right]=\left[0_{\Re} \oplus\left(S_{i j}\right)\right](x \oplus y \oplus z),
\end{aligned}
$$

and our claim is established. Let $G \in \mathcal{L}\left(\Re \otimes C^{n}\right)\left(=\mathscr{L}(\Re) \otimes \Re_{n}\right)$ be the essentially unitary operator given by $G=U^{*} W U \oplus\left(1_{\Re} \otimes 1_{n-1}\right)$. The preceding remark shows that $G\left(S_{i j}\right) G^{*}$ is unitarily equivalent to $0_{\Re} \oplus\left(S_{i j}\right)$ and 
hence unitarily equivalent to the operator $T$. Let $Z=U^{*} W U$ and let $R_{i j}=$ $Z S_{i j} Z^{*}, 1 \leqslant i, j \leqslant n$. Then the families $\left\{R_{i j}: 1 \leqslant i, j \leqslant n\right\}$ and $\left\{S_{i j}: 1 \leqslant i, j \leqslant\right.$ $n$ ) are simultaneously weakly equivalent via the operator $Z$ (notice that $\pi Z$ is unitary in $2(\Re)$ ), and since each of these families consists of essentially commuting essentially normal operators, from [10, Theorem 4.3], it follows that these two families are simultaneously strongly equivalent, i.e. there exists a unitary $Y$ in $\mathcal{L}(\mathcal{C})$ such that $Z S_{i j} Z^{*}-Y S_{i j} Y^{*} \in \mathcal{K}(\mathcal{T}), 1<i, j<n$. In order to complete the proof of the theorem it clearly suffices to prove that the $n \times n$ operator matrix in $\mathcal{L}(\mathcal{C}) \otimes \Re_{n}$ given by

$$
\begin{aligned}
\left(1_{\Re} \oplus\left(Y \otimes 1_{n-1}\right)\right)\left(z \oplus\left(1_{\Re} \otimes 1_{n-1}\right)\right)\left(S_{i j}\right) & \cdot\left(Z \oplus\left(1_{\Re} \otimes 1_{n-1}\right)\right)^{*}\left(1_{\Re} \oplus\left(Y \otimes 1_{n-1}\right)\right)^{*} \\
= & \left(\begin{array}{cccc}
Z S_{11} Z^{*} & Z S_{12} Y^{*} & \cdots & Z S_{1 n} Y^{*} \\
Y S_{21} Z^{*} & Y S_{22} Y^{*} & \cdots & Y S_{2 n} Y^{*} \\
\vdots & \vdots & \vdots \\
Y S_{n 1} Z^{*} & Y S_{n 2} Y^{*} & \cdots & \cdots S_{n n} Y^{*}
\end{array}\right)
\end{aligned}
$$

has essentially commuting essentially normal entries. In order to see that the entries are essentially normal we need only check that, for $1<j<n$, the operators $Z S_{1 j} Y^{*}$ and $Y S_{j 1} Z^{*}$ are essentially normal, and this is a consequence of the fact that $Z S_{1 j} Z^{*}-Y S_{1 j} Y^{*} \in \mathcal{K}(\mathcal{T})$ and $Z S_{j 1} Z^{*}-Y S_{j 1} Y^{*}$ $\in \mathscr{K}(\Re)$. In order to show that the entries of the above matrix essentially commute we use a similar argument. For instance,

$$
\begin{aligned}
& Z S_{11} Z^{*} Y S_{i j} Y^{*}-Y S_{i j} Y^{*} Z S_{11} Z^{*}=Z S_{11} Z^{*} Z S_{i j} Z^{*}-Z S_{i j} Z^{*} Z S_{11} Z^{*} \\
& +Z S_{11} Z^{*}\left(Y S_{i j} Y^{*}-Z S_{i j} Z^{*}\right)-\left(Y S_{i j} Y^{*}-Z S_{i j} Z^{*}\right) Z S_{11} Z^{*} \in \mathcal{K}(\Re),
\end{aligned}
$$

for $1<i, j<n$. The proof of the theorem is complete.

2. The algebra $C_{x}^{*}(X)$. In this section we investigate some properties of hypoconvex sets which will be needed in the next section, and which seems to be useful for the classification problem of essentially $n$-normal operators.

We first introduce some terminology. Throughout, unless otherwise specified, all $C^{*}$-algebras considered will be unital and separable. Given two $C^{*}$-algebras $\mathscr{Q}$ and $\mathscr{B}, \operatorname{Hom}(\mathcal{Q}, \mathscr{B})$ denotes the set of all unital *-homomorphisms from $\mathbb{Q}$ into $\mathscr{B}$. Given a compact Hausdorff space $Z$, the $C^{*}$-algebra $C(Z) \otimes \Re_{n}$ will be identified with the $C^{*}$-algebra of all $n \times n$ continuous matrix valued functions on $Z$. Also, given a compact subset $X$ of $\Re_{n}$ we denote by $C_{x}^{*}(X)$ [30] the $C^{*}$-subalgebra of $C(X) \otimes \Re_{n}$ generated by the identity function $X: X \rightarrow X$ and the constant function 1 on $X$. Given a sequence $\left\{A_{j}\right\}$ in the $C^{*}$-algebra $\mathcal{Q}, C^{*}\left(\left\{A_{j}\right\}\right)$ will denote the $C^{*}$-subalgebra of $\mathcal{Q}$ generated by $\left\{A_{j}\right\}$ and 1 . The following resembles the definition of the Gelfand transform. 
Definition 2.1. Let $\mathbb{Q}$ be a unital $C^{*}$-algebra and let $A \in \mathbb{Q}$. Also, let $R^{n}(A)=\left\{L \in \mathfrak{N}_{n}\right.$ : there exists $\varphi \in \operatorname{Hom}\left(C^{*}(A), \mathfrak{N}_{n}\right)$ such that $\left.\varphi A=L\right\}$. Let $\Gamma_{A}: C^{*}(A) \rightarrow C_{\chi}^{*}\left(R^{n}(A)\right)$ be defined as follows: given $L \in R^{n}(A)$, let $\varphi_{L} \in \operatorname{Hom}\left(C^{*}(A), \mathfrak{N}_{n}\right)$ be the *-homomorphism determined by $\varphi_{L} A=L$. Then, for each $B \in C^{*}(A)$ we define $\Gamma_{A} B(L)=\varphi_{L} B$.

REMARK 2.2. It is easy to check that $\Gamma$ is a surjective *-homomorphism, that is a *-isomorphism if $\bigcap_{\varphi \in \operatorname{Hom}\left(C^{*}(A), 9 \pi_{n}\right)} \operatorname{ker} \varphi=\{0\}$. This occurs, for instance, if $n=1$ and $A$ is normal, in which case $\Gamma_{A}$ coincides with the Gelfand transform, which provides for the continuous functional calculus of $C^{*}(A)$. Our purpose is to make use of the generalized continuous functional calculus provided by the map $\Gamma_{A}$ of Definition 2.1 in order to study certain properties of the $C^{*}$-algebra $C_{X}^{*}(X)$.

LEMMA 2.3. Let $\mathbb{Q}=\mathscr{B} \otimes \Re_{n}$, where $\mathscr{B}$ is a unital abelian $C^{*}$-algebra, and let $A \in \mathbb{Q}$. Then $\Gamma_{A}: C^{*}(A) \rightarrow C_{\chi}^{*}\left(R^{n}(A)\right)$ is a *-isomorphism. Furthermore, if we identify $A$ with a matrix $\left(B_{i, j}\right)$, with entries $B_{i, j}$ in $\mathscr{B}, 1<i, j<n$, and $Y \subset \mathfrak{R}_{n}$ is defined as $Y=\left\{L \in \mathfrak{N}_{n}\right.$ : there exists a character $\omega$ of $\left.C^{*}\left(\left\{B_{i, j}\right\}\right),\left(\omega B_{i, j}\right)=L\right\}$, then $\hat{Y}=R^{n}(A)$.

Proof. To prove the first assertion we need only show that $\bigcap_{\varphi \in \operatorname{Hom}\left(C^{*}(A), \mathscr{2} R_{n}\right)} \operatorname{ker} \varphi=\{0\}$. Thus, we let $A^{\prime} \in C^{*}(A), A^{\prime} \neq 0$, and we identify $A^{\prime}$ with the $n \times n$ matrix $\left(B_{i, j}^{\prime}\right)$, where $B_{i, j}^{\prime} \in C^{*}\left(\left\{B_{i, j}\right\}\right), 1<i, j<n$. Then, since some $B_{k, l}^{\prime} \neq 0$ for $1<k, l<n$, we can find a character $\omega$ of $C^{*}\left(\left\{B_{i, j}\right\}\right)$ such that $\omega B_{k, l}^{\prime} \neq 0$. Now, if we let $L=\left(\omega B_{i, j}\right)$, it follows that $\varphi_{L} A^{\prime} \neq 0$. To prove the last statement, let $L \in R^{n}(A)$ and write $L$, on some basis of $\mathbf{C}^{n}$, as

(***) $L=\sum_{k=1}^{m} \oplus L_{k}$, where $L_{k}$ is an irreducible matrix in $\Re_{n_{k}}, \sum_{k=1}^{m} n_{k}$ $=n, 1<k \leqslant m$.

Let $\psi_{k}: C^{*}(A) \rightarrow \mathfrak{N}_{m_{k}}$ be the irreducible representation uniquely determined by $\psi_{k} A=L_{k}, 1 \leqslant k \leqslant m$. From [17, Theorem 2.10.2], there exists a dilation $\varphi_{k} \in \operatorname{Hom}\left(C^{*}\left(\left\{B_{i, j}\right\}\right) \otimes \Re_{n}, \Re_{n}\right)$ of $\psi_{k}$ such that $\varphi_{k} A=L_{k} \oplus L_{k}^{\prime}$, for some $L_{k}^{\prime} \in \Re_{n-n_{k}}, 1 \leqslant k \leqslant m$. Furthermore, it follows that there exists a character $\omega_{k}$ of $C^{*}\left(\left\{B_{i, j}\right\}\right)$ such that $\varphi_{k}\left(B_{i, j}^{\prime}\right)=\left(\omega_{k} B_{i, j}^{\prime}\right)$, for every $\left(B_{i, j}^{\prime}\right) \in$ $C^{*}\left(\left\{B_{i j}\right\}\right) \otimes \Re_{n}$. In particular, $\left(\omega_{k} A\right)=L_{k} \oplus L_{k}^{\prime}, 1<k<m$, and the proof of the lemma is complete.

COROLlaRY 2.4. (a) Let $Z$ be a compact Hausdorff space and let $f \in C(Z)$ $\otimes \mathfrak{T}_{n}$. Then $C^{*}(f)$ is *-isomorphic to $C_{x}^{*}\left([\text { range } f]^{*}\right)$.

(b) If $Y$ is a nonempty compact subset of $\mathfrak{T}_{n}$, then, via the inclusion map $Y \rightarrow \hat{Y}, C_{\chi}^{*}(Y)$ is *-isomorphic to $C_{\chi}^{*}(\hat{Y})$.

Proof. It is immediate from Lemma 2.3.

In the following lemma we list some functorial properties of hypoconvexity. 
LEMMA 2.5. Let $X, Y$ be two nonempty compact subsets of $\mathbb{R}_{n}$.

(a) Let $f \in C_{\chi}^{*}(X)$ such that range $f \subset Y$ and define $f^{*}: C_{x}^{*}(Y) \rightarrow C_{\chi}^{*}(X)$ by $f^{*} h=h \circ f$, for $h \in C_{\chi}^{*}(Y)$. Then $f^{*}$ is a unital *-homomorphism. Furthermore, if $g \in C_{x}^{*}(Y)$, then $g \circ f \in C_{x}^{*}(X)$ and $(g \circ f)^{*}=f^{*} g^{*}$.

(b) If $Y$ is hypoconvex, $Z$ is a compact Hausdorff space, $\rho \in$ $\operatorname{Hom}\left(C_{\chi}^{*}(Y), C(Z) \otimes \Re_{n}\right)$ and $f=\rho \chi$, then range $f \subset Y$ and $\rho=f^{*}$, where $f^{*}$ is defined as in (a).

(c) If $X$ and $Y$ are hypoconvex, $\rho: C_{\chi}^{*}(Y) \rightarrow C_{\chi}^{*}(X)$ is a *-isomorphism and $f=\rho \chi$, then $f$ is bijective, $C^{*}(f)=C_{\chi}^{*}(X), f^{-1}: Y \rightarrow X$ is in $C_{\chi}^{*}(Y)$, and $\left(f^{-1}\right)^{*}=\rho^{-1}$.

Proof. Part (a) is easy and the only thing that needs some checking in part (b) is the fact that range $f \subset Y$. To this end, let $z \in Z$ and let $\varphi \in$ $\operatorname{Hom}\left(C_{x}^{*}(Y), \mathfrak{T}_{n}\right)$ given by $\varphi h=\rho h(z), h \in C_{x}^{*}(Y)$. On some basis of $\mathbf{C}^{n}$, we decompose $\varphi$ in its irreducible subrepresentations $\varphi=\sum_{k=1}^{m} \oplus \varphi_{k}$, where $\varphi_{k}: C_{x}^{*}(Y) \rightarrow \Re_{n_{k}}, 1 \leqslant k \leqslant m, \sum_{k=1}^{m} n_{k}=n$. Employing [17, Theorem 2.10.2], we deduce that for each $k=1,2, \ldots, m$, there exists an irreducible representation $\psi_{k}: C(Y) \otimes \mathfrak{N}_{n} \rightarrow \mathfrak{N}_{n}$, that satisfies $\psi_{k} h=\varphi_{k} h \oplus \varphi_{k}^{\prime} h$ for every $h \in C_{\chi}^{*}(Y)$ and some $\varphi_{k}^{\prime} \in \operatorname{Hom}\left(C_{\chi}^{*}(Y), \mathfrak{N}_{n-n_{k}}\right)$. It follows that there exists $M_{k} \in Y$ such that, for every $h \in C(Y) \otimes \Re_{n}, \psi_{k} h=h\left(M_{k}\right), 1<k<m$. In particular, $M_{k}=\chi M_{k}=\varphi_{k} \chi \oplus \varphi_{k}^{\prime} \chi$. Since $Y$ is hypoconvex, it follows that $\varphi \chi=\sum_{k=1}^{m} \varphi_{k} \chi \in Y$, and hence $f(z)=\rho \chi(z)=\varphi \chi \in Y$. Now, the validity of statement (b) clearly follows. In order to prove part (c) let $f=\rho \chi$ and $g=\rho^{-1} \chi$ which lie in $C_{\chi}^{*}(Y)$ and $C_{\chi}^{*}(X)$, respectively. In order to complete the proof of the lemma it suffices to show that $g \circ f=\chi_{X}$ and $f \circ g=\chi_{Y}$ (we check only the first one since the other one follows by symmetry). Let $p_{j}\left(\chi_{Y}, \chi_{Y}^{*}\right), j=1,2, \ldots$, be a sequence of polynomials on the noncommutative variables $\chi_{Y}$ and $\chi_{Y}^{*}$ that converges to $g$. Then

$$
\chi_{X}=\rho \rho^{-1} \chi_{X}=\rho g=\lim _{j \rightarrow \infty}\left(\chi_{Y}, \chi_{Y}^{*}\right)=\lim _{j \rightarrow \infty} p_{j}\left(f, f^{*}\right)=g \circ f .
$$

Notice that this also proves that $\chi_{X} \in C^{*}(f)$. The proof of the lemma is complete.

Defintion 2.6. For each $n=1,2, \ldots$ we denote by $\mathbf{H C}_{n}$ the category of all hypoconvex subsets of $\Re_{n}$, where $\operatorname{Hom}(X, Y)=\left\{f \in C_{X}^{*}(X)\right.$ : range $f \subset$ $Y\}, X, Y \in \mathbf{H C}_{n}$. Functions in $\operatorname{Hom}(X, Y)$ will be called hypoconvex.

REMARK 2.7. (a) Lemma 2.5 tells us that the correspondence $X \rightarrow C_{\chi}^{*}(X)$ defines a completely faithful contravariant functor from $\mathbf{H C}_{n}$ into the category of unital separable $C^{*}$-algebras all of whose irreducible representations are finite dimensional.

(b) Let $X, Y \in \mathbf{H C}_{n}$ and let $f \in \operatorname{Hom}(X, Y)$. Also, let $L_{k} \in X$ and let $P_{k}$ be a projection in $\Re_{n}$ such that $P_{k} L_{k}=L_{k} P_{k}, 1<k<m$, and such that $\sum_{k=1}^{m} P_{k}=1$. Then $P_{k} f\left(L_{k}\right)=f\left(L_{k}\right) P_{k}$ and $f\left(\sum_{k=1}^{m} P_{k} L_{k}\right)=\sum_{k=1}^{m} P_{k} f\left(L_{k}\right)$. 
Therefore, if $X^{\prime} \subset X$, then $\left[f\left(X^{\prime}\right)^{\wedge}\right]^{\wedge}=\left[f\left(X^{\prime}\right)\right]^{\wedge}$. Furthermore, if $p$ is a polynomial in $\chi$ (or $\left.\chi^{*}\right)$, then $\left[p\left(X^{\prime}\right)\right]^{*}=p\left[\left(X^{\prime}\right)^{\wedge}\right]$. On the other hand, if $f^{*}$ : $C_{\chi}^{*}(Y) \rightarrow C_{\chi}^{*}(X)$ is an isomorphism and $\left(X^{\prime}\right)^{\wedge}=X$, then $\left[f\left(X^{\prime}\right)\right]^{\wedge}=Y$; indeed, $\left[f\left(X^{\prime}\right)\right]^{\wedge}=\left[f\left(X^{\prime}\right)^{\wedge}\right]^{\wedge}=[f(X)]^{\wedge}=\hat{Y}=Y$.

DeFINITION 2.8. From now on we shall denote by $\mathcal{G} \Re_{n}$ the set of irreducible matrices in $\Re_{n}$. Given a hypoconvex $X \subset 9 \Re_{n}$, we say that a compact subset $X^{\prime}$ of $X$ is generating for $X$, if $X^{\prime}$ consists of inequivalent matrices and $\left(X^{\prime}\right)^{\wedge}=X$.

THEOREM 2.9. Let $X$ be a hypoconvex set in $\mathcal{G} \mathbb{R}_{n}$. Then the following statements are equivalent.

(a) There exists a generating subset $X^{\prime}$ for $X$.

(b) There exists a nonempty compact subset $X^{\prime}$ of $X$ such that $C_{X}^{*}(X)$ is *-isomorphic to $C\left(X^{\prime}\right) \otimes \mathfrak{N}_{n}$. $Z$.

(c) $C_{x}^{*}(X)$ is *-isomorphic to $C(Z) \otimes \Re_{n}$ for some compact Hausdorff space

Proof. To see that (a) implies (b), first notice that from Corollary 2.4(b) it follows that $C_{\chi}^{*}(X)$ is *-isomorphic to $C_{\chi}^{*}\left(X^{\prime}\right)$. Now, from the generalized Stone-Weierstrass theorem [17, Lemma 11.1.4] and the fact that $X^{\prime}$ consists of inequivalent irreducible matrices we see that $C_{x}^{*}\left(X^{\prime}\right)=C\left(X^{\prime}\right) \otimes \Re_{n}$. Thus, we have proved that (a) implies (b). Since (b) implies (c) is obvious, we turn to the proof that (c) implies (a). Suppose that $\rho: C_{x}^{*}(X) \rightarrow C(Z) \otimes \Re_{n}$ is a *-isomorphism and let $f=\rho x$. From Lemma $2.5(\mathrm{~b})$ we deduce that range $f$ $\subset X$ and since $C^{*}(f)=C^{*}(\rho \chi)=\rho C_{\chi}^{*}(X)=C(Z) \otimes \Re_{n}$, it follows that range $f$ consists of inequivalent matrices. Let $Y=$ [range $f]^{\circ}$, and let $\sigma$ : $C_{\chi}^{*}(X) \rightarrow C_{\chi}^{*}(Y)$ be the *-isomorphism given by $\sigma=\Gamma_{f} \circ \rho$ where $\Gamma_{f}$ is defined as in Definition 2.1. We deduce that if we set $g=\sigma \chi$, then $g: Y \rightarrow X$ is bijective and $g^{*}=\sigma$. But, $g=\sigma \chi_{X}=\Gamma_{f} \rho \chi_{X}=\Gamma_{f} f=\chi_{Y}$. Since $g$ is bijective $\chi_{Y}=\chi_{X}$, and hence $X=Y$. In particular, [range $\left.f\right]^{\circ}=X$ and therefore range $f$ is a generating set for $X$. The proof of the theorem is complete.

REMARK 2.10. (a) If $X$ is a hypoconvex subset of $G T_{2}$, then there exists a canonical generating set $X \sim$ for $X$. In fact, it follows from [24, Theorem 3.1] that if $\approx$ denotes the relation of unitary equivalence on $\Re_{n}$, and $p_{n}$ : $\Re_{n} \rightarrow \Re_{n} / \approx$ denotes the quotient map, then there exists a continuous cross-section $s: \mathfrak{T}_{2} / \approx \rightarrow \Re_{2}$ of $p_{2}$, i.e., $p_{2} \circ s$ is the identity on $\mathfrak{T}_{2} / \approx$. Thus, we simply set $X \sim=s \circ p_{2}(X)$ for every hypoconvex $X \subset 9 \Re_{2}$.

(b) For $n>2$ in general there are hypoconvex subsets of $\mathscr{G} \Re_{n}$ with no generating subsets for them. Indeed, this is an easy consequence of the proof of [24, Theorem 3.2] where it is shown that there exists no continuous cross-section of the quotient $\operatorname{map}_{p_{n}}: \mathfrak{T}_{n} \rightarrow \mathfrak{T}_{n} / \approx$, for $n>2$.

(c) If $X$ is a hypoconvex subset of $\mathscr{T}_{n}$ which does not consist only of 
irreducible matrices, then it is easy to see that $C_{\chi}^{*}(X)$ cannot be *-isomorphic to $C(Z) \otimes \Re_{n}$, for any compact Hausdorff space $Z$.

We are indebted to Vern Paulsen who pointed out a mistake in an earlier version of the above remark.

3. Extensions of $C^{*}$-algebras. In this section we explain how the classification problem for essentially $n$-normal operators can be rephrased in terms of certain classification problems in the theory of $C^{*}$-algebra extensions of the ideal of compact operators on $\mathcal{H}$. A similar formulation was already used in [10] where extension theory was used to solve the classification problem for essentially normal operators. We now recall some definitions and certain background material that will be needed in the sequel.

For a (unital, separable) $C^{*}$-algebra $\mathscr{Q}$ we let $E(\mathscr{Q})$ be the set of all unital *-monomorphisms $\tau: \mathbb{Q} \rightarrow \mathcal{2}(\mathcal{F})$. We say that $\tau$ and $\tau^{\prime}$ in $E(\mathbb{Q})$ are weakly [resp., strongly] equivalent, and we write $\tau \sim \tau^{\prime}$ [resp., $\tau \sim \sim_{s} \tau^{\prime}$ ] if there exists $W$ in $\mathcal{L}(\mathcal{H C}), \pi W$ unitary [resp., $W$ unitary] such that $\pi W^{*}(\tau A) \pi W=\tau^{\prime} A$ for every $A \in \mathbb{Q}$. For each $\tau$ in $E(\mathbb{Q})$ we denote by $[\tau]$ and $[\tau]_{s}$ the weak and strong equivalence classes containing $\tau$, respectively, and we denote by $\operatorname{Ext}(\mathbb{Q})$ and $\operatorname{Ext}^{\mathrm{s}}(\mathbb{Q})$ the set of all weak equivalence classes and strong equivalence classes of $E(\mathbb{Q})$, respectively. We define the following natural abelian operations on $\operatorname{Ext}(\mathscr{Q})$ and $\operatorname{Ext}^{s}(\mathbb{Q}):[\tau]+\left[\tau^{\prime}\right]=\left[\tau \oplus \tau^{\prime}\right]$ and $[\tau]_{s}+$ $\left[\tau^{\prime}\right]_{s}=\left[\tau \oplus \tau^{\prime}\right]_{s}, \tau$ and $\tau^{\prime}$ in $E(\mathbb{Q})$, where $\tau \oplus \tau^{\prime}: \mathbb{Q} \rightarrow \mathcal{2}(\mathcal{H C}) \oplus \mathscr{2}(\mathcal{H C})$ has been identified with an element of $E(\mathbb{Q})$ via an identification of $2(\mathcal{H}) \oplus$ $\mathcal{2}(\mathcal{H})$ with a $C^{*}$-subalgebra of $\mathcal{2}(\mathcal{H})$. We shall say that $\tau \in E(\mathbb{Q})$ is trivial if there exists $\sigma \in \operatorname{Hom}(\mathbb{Q}, \mathcal{L}(\mathcal{K})$ ) such that $\tau=\pi \sigma$ and the set of all trivial elements in $E(\mathscr{Q})$ will be denoted by $E_{0}(\mathscr{Q})$.

One of the most remarkable generalizations of the work of Brown, Douglas and Fillmore [10] was the discovery ([34] and [10]) that if $Q$ is a nuclear $C^{*}$-algebra then $\operatorname{Ext}(\mathscr{Q})$ and $\operatorname{Ext}^{\Im}(\mathscr{Q})$ form abelian groups whose identities consist of the weak equivalence class and strong equivalence class containing $E_{0}(\mathbb{Q})$, respectively (see also [3]). In [10] it was shown that for every compact metric space $X, \operatorname{Ext}[C(X)]=\operatorname{Ext}^{s}[C(X)]$. For this reason we shall denote by $\operatorname{Ext}(X)$ either of the sets $\operatorname{Ext}[C(X)]$ or $\operatorname{Ext}^{s}[C(X)]$ for a compact metric space $X$. They also showed that $\operatorname{Ext}(\cdot)$ is a homotopy invariant covariant functor from the category of compact metric spaces into the category of abelian groups, and in [31] this result was generalized to the category of quasi-diagonal nuclear separable $C^{*}$-algebras. Given two $C^{*}$-algebras $\mathbb{Q}$ and $\mathscr{B}$ we shall endow $\operatorname{Hom}(Q, \mathscr{B})$ with the strong operator topology. Also, if $\zeta \in$ $\operatorname{Hom}(\mathscr{Q}, \mathscr{B})$ we define $\zeta_{*}: \operatorname{Ext}(\mathscr{B}) \rightarrow \operatorname{Ext}(\mathscr{Q})$ and $\zeta_{*}^{s}: \operatorname{Ext}^{s}(\mathscr{B}) \rightarrow \operatorname{Ext}^{s}(\mathscr{Q})$ by $\zeta_{*}[\tau]=\left[(\tau \circ \zeta) \oplus \tau_{0}\right]$ and $\zeta_{*}^{s}[\tau]_{s}=\left[(\tau \circ \zeta) \oplus \tau_{0}\right]_{s}$, respectively, where $\tau_{0} \in$ $E_{0}(\mathbb{Q})$. One of the corollaries of the main result in [31] is the following homotopy theorem. 
THEOREM 3.1. Let $\mathcal{Q}$ and $\mathscr{B}$ be unital separable $C^{*}$-algebras all of whose irreducible representations are finite dimensional, and let $\theta:[0,1] \rightarrow$ $\operatorname{Hom}(Q, \mathscr{B})$ be a continuous function, then $[\theta(0)]_{*}=[\theta(1)]_{*}$ and $[\theta(0)]_{*}^{*}=$ $[\theta(1)]_{*}^{s}$.

REMARK 3.2. The map $\Phi: \operatorname{Ext}^{s}(\mathbb{Q}) \rightarrow \operatorname{Ext}(\mathbb{Q})$ given by $\Phi[\tau]_{s}=[\tau]$ for $\tau \in E(\mathbb{Q})$ is a surjective homomorphism but in general is not injective. Also, $\Phi$ is a natural transformation from the contravariant functor $\operatorname{Ext}^{5}(\cdot)$ to the contravariant functor $\operatorname{Ext}(\cdot)$ on the category of unital separable $C^{*}$-algebras. As pointed out in [9] there is a natural action of the integers $\mathbf{Z}$ on $\operatorname{Ext}^{5}(\mathbb{Q})$ given by $\alpha n\left([\tau]_{s}\right)=\left[\pi W^{*} \tau \pi W\right]_{s}$, where $W$ has index $n$ and $\pi W$ is unitary in $\mathcal{Q}(\mathcal{H}), n \in \mathbf{Z}$. It follows that $\operatorname{ker} \Phi$, which is the weak equivalence class containing $E_{0}(\mathbb{Q})$, is the orbit of the action $\alpha$ on the neutral element of $\operatorname{Ext}^{s}(\mathbb{Q})$.

The above action $\alpha$ defines a surjection $\eta: Z \rightarrow \operatorname{ker} \Phi$. More precisely, $\eta$ can be defined as follows: Let $\tau_{0} \in E_{0}(\mathscr{Q})$ and let $U^{+}$be a unilateral shift in $\mathcal{L}(\mathcal{H})$; then for $k \in \mathbf{Z}$, we let $\eta(k)=\left[\left(\pi U^{+k}\right) \tau_{0}\left(\pi U^{+* k}\right)\right]$. Since $U^{+k}$ is unitarily equivalent to $U^{+} \oplus U^{+} \oplus \cdots \oplus U^{+}$( $k$-times), it readily follows that $\eta$ is a homomorphism.

The next result (which was announced in [30]) shows that kernel of $\Phi$ can be explicitly computed in certain cases. We are indebted to the referee of this paper for having suggested a simplification in our original proof of the following lemma.

LEMMA 3.3. Let $Q$ be a (unital, separable) $C^{*}$-algebra.

(a) If there exists an $n$-dimensional irreducible representation of $\mathcal{Q}$, then ker $\Phi$ is a homomorphic image of $Z_{n}(=Z / n Z)$.

(b) If there exists a unital inclusion $j: \mathfrak{R}_{n} \rightarrow \mathbb{Q}$, then $\mathbf{Z}_{n}$ is a homomorphic image of ker $\Phi$. In particular, if $\mathbb{Q}=\mathscr{B} \otimes \mathfrak{K}_{n}$, for some abelian $C^{*}$-algebra $\mathscr{B}$, then $\operatorname{ker} \Phi \cong \mathbf{Z}_{n}$.

Proof. Let $\eta: Z \rightarrow \operatorname{ker} \Phi$ be as in the above remark. In order to prove part (a), it clearly suffices to show that $\eta(1)$ has order at most $n$. Thus, let $\sigma_{0}: \mathbb{Q} \rightarrow \mathscr{L}(\mathcal{H})$ be a faithful unital representation so that $\pi \sigma_{0}\left(=\tau_{0}\right) \in E_{0}(\mathbb{Q})$. Also, let $P=1-U^{+n} U^{+* n}$, and let $\varphi: \mathbb{Q} \rightarrow \mathcal{L}(P \mathscr{C})$ be an $n$-dimensional irreducible representation of $\mathbb{Q}$ (notice that $\operatorname{dim}(P \mathscr{C})=n$ ). It easily follows that the map $\sigma: \mathbb{Q} \rightarrow \mathcal{E}(\mathcal{K})$ defined by $\sigma(A)=U^{+n}\left(\sigma_{0} A\right) U^{+\neq n}+(\varphi A) \circ P$ is a unital faithful representation of $\mathbb{Q}$. Moreover,

$$
\begin{aligned}
n \eta(1) & =\eta(n)=\left[\left(\pi U^{+n}\right) \pi \sigma_{0}\left(\pi U^{+* n}\right)\right] \\
& =\left[\pi\left(U^{+n} \sigma_{0} U^{+* n}+\varphi P\right)\right]=[\pi \sigma]=0,
\end{aligned}
$$

as desired. In order to prove (b) we first observe that if $\mathbb{Q}^{\prime}$ is another $C^{*}$-algebra and $\zeta$ in $\operatorname{Hom}\left(Q^{\prime}, \mathscr{Q}\right)$ is injective, then for every $\tau_{0}$ in $E_{0}(\mathscr{Q})$, it 
follows that $\tau_{0} \zeta$ is in $E_{0}\left(\mathbb{Q}^{\prime}\right)$, and hence the following diagram is commutative.

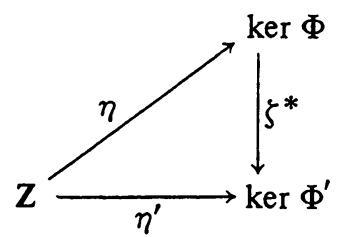

where the definitions of the maps $\Phi^{\prime}$ and $\eta^{\prime}$ are similar to those of $\Phi$ and $\eta$, respectively. The proof of the lemma is completed by applying the preceding remark to the map $\zeta=j$, and the $C^{*}$-algebra $\mathbb{Q}^{\prime}=\mathfrak{N}_{n}$, and observing $\operatorname{ker} \Phi^{\prime}=\operatorname{Ext}^{s}\left(\Re_{n}\right) \approx Z_{n}$ (cf. [19]).

LemMa 3.4. Let $\mathcal{Q}$ be as above and let $n$ be a positive integer. Also, let $\Psi$ : $\operatorname{Ext}(\mathbb{Q}) \rightarrow \operatorname{Ext}\left(\mathbb{Q} \otimes \mathfrak{K}_{n}\right)$ be given by $\Psi[\tau]=\left[\tau \otimes 1_{n}\right]$. Then $\Psi$ is an isomorphism.

Proof. Since it is obvious that $\Psi$ is a homomorphism we prove that $\Psi$ is bijective by exhibiting an inverse $\Psi^{\prime}: \operatorname{Ext}\left(\mathbb{Q} \otimes \mathscr{T}_{n}\right) \rightarrow \operatorname{Ext}(\mathbb{Q})$ of $\Psi$. Let $\tau \in E\left(\mathbb{Q} \otimes \Re_{n}\right)$ and let $\left\{e_{i, j}: 1 \leqslant i, j \leqslant n\right\}$ be the natural system of matrix units in $\mathcal{Q} \otimes \mathfrak{N}_{n}$. Then, $\left\{\tau e_{i, j}: 1<i, j<n\right\}$ is a system of matrix units in $\mathcal{Q}(\mathcal{H C})$ such that $\sum_{i=1}^{n} \tau e_{i, i}=1$. Reasoning, for instance, as in [26, Lemma 3.1], there exists a system of matrix units $\left\{W_{i, j}: 1<i, j<n\right\}$ in $\mathcal{L}(\mathcal{H C})$ such that $\pi W_{i, j}=\tau e_{i, j}$ and the subspace $\mathcal{H}^{\prime}=$ range $\sum_{i=1}^{n} W_{i, i}$ has codimension less than $n$ in $\mathcal{H}$. Now, let $V$ be any isometry in $\mathcal{L}(\mathcal{K})$ such that range $V=\mathcal{X}^{\prime}$.

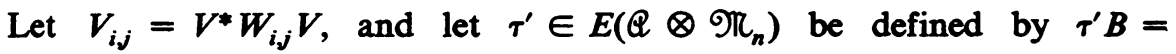
$\pi V^{*} \tau B \pi V, B \in \mathbb{Q} \otimes \Re_{n}$. We deduce that $\left\{V_{i, j}\right\}$ is a system of matrix units in $\mathcal{E}(\mathcal{F})$ such that $\sum_{i=1}^{n} V_{i, i}=1_{\mathcal{O}}$ and $\tau^{\prime} e_{i, j}=\pi V_{i, j}, 1<i, j<n$. It readily follows that there exists a unitary transformation $U: \mathcal{H} \rightarrow \mathcal{H} \otimes C^{n}$ such that $U V_{i, j} U^{*}$ is the $n \times n$ operator matrix in $\mathcal{L}\left(\mathcal{K} \otimes \Re_{n}\right)$, all of whose entries are zero except the $(i, j)$ th entry which is $1_{\mathscr{X}}$. Via the unitary $U$, the *-monomorphism $\tau^{\prime}: \mathbb{Q} \otimes \mathscr{T}_{n} \rightarrow \mathcal{Q}(\mathcal{H})$ can be identified with a *-monomorphism, which we still call $\tau^{\prime}$, from $Q \otimes \Re_{n}$ into $2(\mathscr{T}) \otimes \mathfrak{N}_{n}$. We define now the positive linear map $\tilde{\tau}: \mathbb{Q} \rightarrow \mathcal{2}(\mathcal{H})$ as follows: For each $A \in \mathbb{Q}$, we let $\tilde{\tau} A$ be the 1,1 entry of the matrix in $\mathcal{2}(\mathcal{F}) \otimes \Re_{n}$ given by $\tau^{\prime}\left(A \otimes e_{1,1}\right)$. In view of the above identifications, it follows that $\tilde{\tau}$ is a unital *-monomorphism, and that

$$
\begin{aligned}
\tau^{\prime}\left(A \otimes e_{i, j}\right) & =\tau^{\prime}\left(\left(1 \otimes e_{i, 1}\right)\left(A \otimes e_{1,1}\right)\left(1 \otimes e_{1, j}\right)\right) \\
& =\left(1 \otimes e_{i, 1}\right)\left((\tilde{\tau} A) \otimes e_{1,1}\right)\left(1 \otimes e_{1, j}\right)=(\tilde{\tau} A) \otimes e_{i, j},
\end{aligned}
$$

$1<i, j<n, A \in \mathbb{Q}$. Therefore, given $\left(A_{i j}\right)$ in $\mathbb{Q} \otimes \mathfrak{T}_{n}$, we have $\tau^{\prime}\left(A_{i j}\right)=$ $\sum_{i, j} \tau^{\prime}\left(A_{i j} \otimes e_{i, j}\right)=\Sigma_{i j}\left(\tilde{\tau} A_{i j}\right) \otimes e_{i, j}=\left(\tilde{\tau} \otimes 1_{n}\right)\left(A_{i j}\right)$, that is, $\tau^{\prime}=\tilde{\tau} \otimes 1_{n}$. Suppose now that $\tilde{\tau} \otimes 1_{n} \sim \tau_{1} \otimes 1_{n}$ for some $\tau_{1}$ in $E(\mathbb{Q})$. This means that 
there exists an essentially unitary $W$ in $\mathcal{E}(\mathscr{T C}) \otimes \mathbb{N}_{n}$ such that $(\pi W)\left(\tilde{\tau} \otimes 1_{n}\right)$ $=\left(\tau_{1} \otimes 1_{n}\right)(\pi W)$. In particular, $(\pi W)\left(1 \otimes e_{i, j}\right)=\left(1 \otimes e_{i, j}\right)(\pi W), 1<i, j<n$. It readily follows that there exists an essentially unitary $W^{\prime}$ in $\mathcal{E}(\mathcal{F C})$ such that $W=W^{\prime} \otimes 1_{n}$, and $\left(\pi W^{\prime}\right) \tilde{\tau}=\tau_{1}\left(\pi W^{\prime}\right)$, and hence $\tilde{\tau} \sim \tau_{1}$. Now we define the map $\Psi^{\prime}$ by letting $\Psi^{\prime}[\tau]\left(=\Psi^{\prime}\left[\tau^{\prime}\right]\right)=[\tilde{\tau}]$. The above remarks show that $\Psi^{\prime}$ is well defined and it is the inverse of $\Psi$, as required.

LEMMA 3.5. Let $X$ be a nonempty compact subset of $\mathfrak{T}_{n}$. If $\tau \in E(C(X) \otimes$ $\Re_{n}$ ) and $T$ is an operator in $\mathcal{L}(\mathcal{F})$ such that $\pi T=\tau \chi$, then $T$ is essentially $n$-normal and $R_{e}^{n}(T)=\hat{X}$. Conversely, if $X$ is hypoconvex and $T$ is any essentially n-normal operator such that $R_{e}^{n}(T)=X$, then there exists $a \tau$ in $E\left(C(X) \otimes \pi_{n}\right)$ such that $\tau \chi=\pi T$.

Proof. In order to prove the first part of the theorem, we let $\tau^{\prime}: C(X) \otimes$ $\Re_{n} \rightarrow \mathcal{2}(\mathcal{H}) \otimes \Re_{n}$, and $\tilde{\tau}: C(X) \rightarrow \mathcal{2}(\mathscr{H})$ be the unital *-monomorphisms induced by $\tau$, as in the proof of Lemma 3.4, where $Q=C(X)$, so that $\tau^{\prime}=\tilde{\tau} \otimes 1_{n}$ and $\tau^{\prime} \sim \tau$. Also let $T^{\prime}$ in $\mathcal{L}(\mathcal{I C})$ be such that $\pi T^{\prime}=\tau^{\prime} \chi$. Then, writing $\chi$ as the $n \times n$ matrix $\left(\chi_{i j}\right)$ in $C(X) \otimes \Re_{n}$, and representing $T^{\prime}$ as the $n \times n$ matrix $\left(T_{i j}^{\prime}\right)$ in $\mathcal{L}(\mathcal{H C}) \otimes \pi_{n}$, we also have $\tilde{\tau}_{i j}=\pi_{\mathscr{C}} T_{i j}^{\prime}, 1<i, j<n$. Thus $\left(\pi T_{i j}^{\prime}\right)$ is an $n \times n$ matrix of commuting normal entries in $2(\mathcal{F})$, and hence $T^{\prime}$ is an essentially $n$-normal operator in $\mathcal{L}\left(\mathcal{H} \otimes C^{n}\right)=\mathscr{L}(\mathcal{H}) \otimes \pi_{n}$. Let $\mathscr{B}=C^{*}\left(\left\{\pi T_{i j}^{\prime}\right\}\right)$. Then $\pi T^{\prime}$ is an element of $\mathscr{B} \otimes \pi_{n}$, and from Lemma $2.3 \Gamma_{\pi T^{\prime}}$ is a *-isomorphism from $C^{*}\left(\pi T^{\prime}\right)$ onto $C_{x}^{*}\left(R^{n}\left(\pi T^{\prime}\right)\right)$. Notice that $R^{n}\left(\pi T^{\prime}\right)=R_{e}^{n}\left(T^{\prime}\right)$ and that the hypoconvex hull of the joint essential spectrum of $T_{i j}^{\prime}$ coincides with $R_{e}^{n}\left(T^{\prime}\right)$ (cf. Lemma 2.3). Let $\rho: C^{*}\left(\pi T^{\prime}\right) \rightarrow$ $C_{x}^{*}(X)$ be the restriction of $\left(\tau^{\tau^{-1}}\right)^{-1}$ to $C^{*}\left(\pi T^{\prime}\right)$ and let $L \in X$. Then the map $A \rightarrow \rho A(L), A \in C^{*}\left(\pi T^{\prime}\right) \quad\left(=C^{*}\left(\tau^{\prime} \chi\right)\right)$ defines a homomorphism in $\operatorname{Hom}\left(C^{*}\left(\pi T^{\prime}\right), \Re_{n}\right)$ whose value at $\pi T^{\prime}$ is $L$, and hence $L \in R_{e}^{n}(T)$. Therefore $X \subset R_{e}^{n}\left(T^{\prime}\right)$ and since $C_{x}^{*}(X)$ and $C_{x}^{*}\left(R_{e}^{n}\left(T^{\prime}\right)\right)$ are isomorphic, from Lemma 2.5 it follows that $\hat{X}=R_{e}^{n}\left(T^{\prime}\right)$. Furthermore, from the fact that $\tau^{\prime} \sim \tau$, and Theorem 1.14, we deduce that if $T$ is any operator in $\mathcal{L}(\mathcal{F C})$ such that $\pi T=\tau \chi$, then $T \sim T^{\prime}$, so that $T$ is essentially $n$-normal and $R_{e}^{n}(T)$ $\left(=R_{c}^{n}\left(T^{\prime}\right)\right)=\hat{X}$. In order to prove the second part of the theorem, suppose that $X$ is hypoconvex and that $T$ is any essentially $n$-normal operator in $\mathcal{L}(\mathcal{H C})$ such that $R_{e}^{n}(T)=X$. Also let $U: \mathcal{K} \rightarrow \mathcal{X} \otimes C^{n}$ be a unitary transformation such that $T^{\prime}=U T U^{*}$ can be identified with an $n \times n$ matrix in $\mathcal{L}(\mathcal{H}) \otimes \mathbb{R}_{n}$ whose entries $T_{i j}^{\prime}$ are essentially commuting, essentially normal operators in $\mathcal{L}(\mathcal{S C})$. Notice that $R_{e}^{n}(T)=R_{e}^{n}\left(T^{\prime}\right)$. It follows that $C^{*}\left(\pi T^{\prime}\right)$ is a $C^{*}$-subalgebra of $C^{*}\left(\left\{\pi T_{i j}^{\prime}\right\}\right) \otimes \mathfrak{N}_{n}$. Let $Y \subseteq \mathbb{N}_{n}$ be the joint essential spectrum of $T_{i j}^{\prime}$, $1<i, j<n$. Then, via the Gelfand transform(cf. Lemma 2.3), $C(Y)$ is *-isomorphic with $C\left(\left\{T_{i j}^{\prime}\right\}\right)$ and hence $\Gamma_{\pi T^{\prime}}^{-1}$ extends to a *-isomorphism $\rho: C(Y) \otimes \pi_{n}$ $\rightarrow C\left(\left\{\pi T_{i j}^{\prime}\right\}\right) \otimes \Re_{n}$. We know, from Lemma 2.3 , that $Y \subset X$ and $\hat{Y}=X$, so 
that the inclusion map $Y \rightarrow X$ induces a homomorphism $\theta: C(X) \otimes \Re_{n} \rightarrow$ $C(Y) \otimes \mathfrak{N}_{n}$ whose restriction to $C_{X}^{*}(X)$, which we denote by $\mathcal{T}$, is a *-isomorphism onto $C_{\chi}^{*}(Y)$ such that $\widetilde{T}_{\chi}=\chi$. Given a trivial extension $\tau_{0}$ in $E_{0}\left(C(X) \otimes \Re_{n}\right)$, let $\tau_{1}$ be the restriction of $\tau_{0}$ to $C_{\chi}^{*}(X)$, and let $\tau^{\prime}=\rho \circ \theta \oplus$ $\tau_{0}$. Then $\tau^{\prime} \in E\left(C(X) \otimes \Re_{n}\right)$ and $\tau^{\prime} / C_{x}^{*}(X)=\rho \circ \mathcal{T} \bigoplus \tau_{1}$. Since $\rho \circ \mathcal{T} \oplus \tau_{1}$ $\sim_{s} \rho \circ \mathcal{T}$, there exists a unitary $V$ in $\mathcal{E}(\mathcal{C})$ such that $\pi V^{*}\left(\rho \circ \mathcal{T}_{X} \oplus \tau_{1} X\right) \pi V$ $=\rho \circ T_{\chi}=\pi T^{\prime}$, so that if we define $\tau$ in $E\left(C(X) \otimes \Re_{n}\right)$ by $\tau A=$ $\pi U^{*} V^{*} \tau^{\prime} A \pi V U$ we obtain an extension of $C(X) \otimes \Re_{n}$ with the required properties.

Employing arguments similar to those given in the above proof we can obtain the following corollary, which gives an alternative proof of Corollary 1.12 .

COROLLARY 3.6. Let $X$ be a hypoconvex subset of $\mathfrak{T}_{n}$. Then there exists a faithful unital representation $\rho: C_{x}^{*}(X) \rightarrow \mathcal{E}(\mathcal{H})$, such that $\rho\left[C_{x}^{*}(X)\right] \cap \mathcal{K}(\mathcal{S C})$ $=\{0\}, \rho \chi$ is n-normal and $R^{n}(\rho \chi)=R_{e}^{n}(\rho \chi)=X$. Indeed, $\rho$ can be taken to be the restriction to $C_{X}^{*}(X)$ of any faithful unital representation $\sigma: C(X) \otimes \pi_{n} \rightarrow$ $\mathcal{L}(\mathcal{H C})$, such that $\sigma\left(C(X) \otimes \mathbb{R}_{n}\right) \cap \mathcal{K}(\mathcal{H})=\{0\}$. On the other hand, if $S$ is an n-normal operator in $\mathcal{L}(\mathcal{H C})$ such that $R^{n}(S)=R_{e}^{n}(S)=X$, then there exists a unital faithful representation $\sigma: C(S) \otimes \Re_{n} \rightarrow \mathcal{L}(\mathcal{H})$ such that $\sigma(C(X) \otimes$ $\left.\mathscr{T}_{n}\right) \cap \mathscr{K}(\mathcal{K})=\{0\}$, and $\sigma \chi=S$.

REMARK 3.7. Given a hypoconvex subset $X$ of $\mathfrak{R}_{n}$, let $\Xi_{X}: \operatorname{Ext}(C(X) \otimes$ $\left.\mathscr{T}_{n}\right) \rightarrow \operatorname{Ext}\left(C_{X}^{*}(X)\right)$, and $\Xi_{x}^{s}: \operatorname{Ext}^{s}\left(C(X) \otimes \Re_{n}\right) \rightarrow \operatorname{Ext}^{s}\left(C_{X}^{*}(X)\right)$ be the maps induced by restricting the elements of $E\left(C(X) \otimes \Re_{n}\right)$ to the $C^{*}$-subalgebra $C_{X}^{*}(X)$. In view of Lemma 3.5 it follows that $[\tau] \in$ range $\Xi_{X}$ if and only if there exists [T] in $E N_{n}(X)$ such that $\pi T=\tau \chi$ (a similar statement holds for $E N_{n}^{s}(X)$ and $\left.\Xi_{X}^{s}\right)$. Let $\nu$ : range $\Xi_{X} \rightarrow E N_{n}(X), \mu$ : range $\Xi_{X}^{s} \rightarrow E N_{n}^{s}(X)$ be the maps defined by $\nu[\tau]=[T], \mu[\tau]_{s}=[T]_{s}$ where $\pi T=\tau \chi$. From the above observation, it follows that $\nu$ and $\mu$ are well-defined isomorphisms. Following [30], we denote by $\operatorname{Ext}_{X}^{n}(X)$, the subgroup range $\Xi_{X}$ of $\operatorname{Ext}\left(C_{X}^{*}(X)\right)$.

The original proof of the following theorem, which was announced in [30], was based on the dilation method of [2] and the main result of [1]. Presently, it is an immediate consequence of the above remark and the fact that $\operatorname{Ext}\left(C(X) \otimes \Re_{n}\right)$ and $\operatorname{Ext}^{s}\left(C(X) \otimes \Re_{n}\right)$ are groups [see [11]].

THEOREM 3.8. Let $X$ be a hypoconvex subset of $\mathfrak{T}_{n}$. Then $E N_{n}(X)$ and $E N_{n}^{s}(S)$ are groups. Furthermore, the map $\beta_{x}=\nu \circ \Xi_{x} \circ \psi(\psi$ is as in Lemma 3.4) is a surjective homomorphism from $\operatorname{Ext}(X)$ to $E N_{n}(X)$.

The following theorem can be used to effectively compute $E N_{n}(X)$ for an important class of hypoconvex sets. 
THEOREM 3.9. Let $X$ be a hypoconvex subset of $9 \Re_{n}$. If $X$ has a generating subset $Y$, then there exists a natural isomorphism $\lambda: \operatorname{Ext}(Y) \rightarrow E N_{n}(X)$, and the kernel of the map $\theta: E N_{n}^{s}(X) \rightarrow E N_{n}(X)$ coincides with $\mathbf{Z}_{n}$.

Proof. We first show that range $\Xi_{X}=\operatorname{Ext}\left(C_{X}^{*}(X)\right)$ and range $\Xi_{X}^{s}=$ $\operatorname{Ext}^{*}\left(C_{X}^{*}(X)\right)$. Since $\hat{Y}=X$, as in the proof of Lemma 3.5, the inclusion map $Y \rightarrow X$ induces a *-homomorphism $\theta: C(X) \otimes \mathbb{R}_{n} \rightarrow C(Y) \otimes \mathfrak{R}_{n}$ whose restriction to $C_{x}^{*}(X)$, which we denote by $\zeta$, is a *-isomorphism onto $C_{x}^{*}(Y)$. From Theorem 2.9 we know that $C_{x}^{*}(Y)=C(Y) \otimes \Re_{n}$ and we deduce that the following diagrams are commutative:

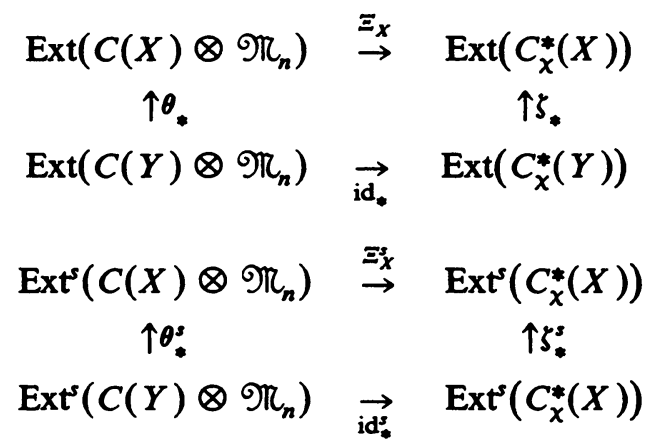

Since $\zeta_{*}$ and $\zeta_{*}^{s}$ are isomorphisms, the map $\Xi_{X}$ and $\Xi_{X}^{s}$ are onto and our claim is established. In particular, the map $\nu$ (introduced in Remark 3.7) is an isomorphism from $\operatorname{Ext}\left(C_{x}^{*}(X)\right)=\operatorname{Ext}_{x}^{n}(X)$ onto $\operatorname{EN}_{n}(X)$. Let $\lambda=\nu \circ \zeta_{*} \circ \Psi$, where $\Psi$ is as in Lemma 3.4. Then $\lambda$ is an isomorphism from $\operatorname{Ext}(Y)$ onto $E N_{n}(X)$. Now the proof of the theorem is completed by using Lemma 3.3, and observing that the following diagram is commutative:

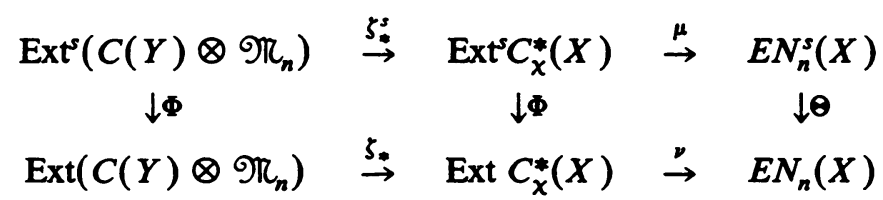

REMARK 3.10. The above theorem allows us to apply the machinery developed in [11] and [22]. In particular, if $Y$ is homotopically equivalent to a subset of the plane, then $E N_{n}(X)$ is isomorphic to the first cohomology group of $Y$, and if $Y$ is homotopically equivalent to a subset of $C^{2}$, then $E N_{n}(X)$ is isomorphic to the direct sum of the first Steenrod homology group of $Y$ with the third Steenrod homology group of $Y$.

Definition 3.11. Given $T$ in $\mathcal{L}(\mathcal{H})$ suppose that $\bigcap_{\varphi \in \operatorname{Hom}\left(C^{*}(\pi T), R_{n}\right)} \operatorname{ker} \varphi$ $=\{0\}$. For each $f \in C_{\chi}^{*}\left[R_{e}^{n}(T)\right]$ we define $f(\pi T)=\Gamma_{\pi T}^{-1} f$, where $\Gamma_{\pi T}$ is defined as in Definition 2.1 (note that we then have the spectral mapping formula: $R^{n}[f(\pi T)]=\left(f\left[R_{e}^{n}(T)\right)^{\wedge}\right)$. Also, given a nonempty compact subset $X$ of $\Re_{n}$ we denote by $\Pi_{\chi}^{n}(X)$ the set of all homotopy classes of invertible 
elements in $C_{\chi}^{*}(X)$ (notice that $\Pi_{\chi}^{n}(X)$ has a natural group structure since it is isomorphic to the index group of $C_{x}^{*}(X)$ [19, Chapter 2]). Furthermore, let $\iota_{X}: E N_{n}(X) \rightarrow \operatorname{Hom}\left(\Pi_{\chi}^{n}(X), \mathbf{Z}\right)$ be the homomorphism given by $\left(\iota_{X}[T]\right)[f]=$ ind $f(\pi T)$, for every $[f] \in \Pi_{\chi}^{n}(X),[T] \in E N_{n}(X)[30]$.

THEOREM 3.12. Let $X$ be a hypoconvex subset of $\mathscr{G} \mathfrak{R}_{n}$ and suppose that $X$ has a generating subset $Y$ which is homotopically equivalent to a compact subset of C. Then, $\iota_{X}$ is an isomorphism. In particular, if $\left\{\left[f_{j}\right] ; j=1,2, \ldots\right\}$ is a system of generators for $\Pi_{\chi}^{n}(X)$, then two essentially n-normal operators $T_{1}$ and $T_{2}$ in $\mathcal{L}(\mathcal{H})$ such that $R_{e}^{n}\left(T_{j}\right)=X$ are weakly equivalent if and only if ind $f_{j}\left(\pi T_{1}\right)=$ ind $f_{j}\left(\pi T_{2}\right)$ for $j=1,2, \ldots$

Proof. As in Theorem 3.9 we observe that Ext $C_{x}^{*}(X)=\operatorname{Ext}_{x}^{n}(X)$, so that $\nu$ is an isomorphism from $\operatorname{Ext}_{\chi}^{*}(X) \rightarrow E N_{n}(X)$ and if we define the homomorphism $\kappa$ : Ext $C_{\chi}^{*}(X) \rightarrow \operatorname{Hom}\left(\Pi_{\chi}^{n}(X), Z\right)$ by $\kappa[\tau][f]=$ ind $\tau f$ where $f$ is an invertible element of $C_{\chi}^{*}(X)$, then the following diagram is easily seen to be commutative:

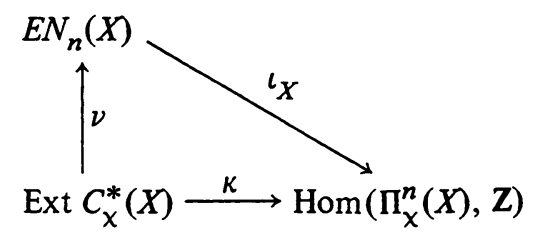

Again, as in the proof of Theorem 3.9, we let $\zeta: C_{x}^{*}(X) \rightarrow C_{x}^{*}(Y)(=C(Y) \otimes$ $\Re_{n}$ ) be the *-isomorphism induced by the inclusion $Y \rightarrow X$. Therefore, $\zeta$ induces an isomorphism $\bar{\zeta}: \Pi_{\chi}^{n}(X) \rightarrow \Pi_{\chi}^{n}(Y)$ so that, by duality, we have an isomorphism $\bar{\zeta}^{*}: \operatorname{Hom}\left(\Pi_{\chi}^{n}(Y), Z\right) \rightarrow \operatorname{Hom}\left(\Pi_{\chi}^{n}(X), Z\right)$. As $\Pi_{\chi}^{n}(Y)$ is the index group of $C(Y) \otimes \Re_{n}$, it can be naturally identified with the set $\left[Y, \mathcal{U}_{u}\right]$ of homotopy classes from $Y$ into the unitary group $\mathcal{U}_{n}$ in $\pi_{n}$. Since $Y$ is homotopically equivalent to a subset of the plane, we can use a standard argument with the homotopy sequence of the fibration $\mathcal{U}_{k} \rightarrow{\mathcal{U}_{k+1}} \rightarrow S^{2 k+1}$ (where $S^{2 k+1}$ is the $2 k+1$ st sphere) (see [32, Chapter 7, §2], [4, p. 239], [6, $\S 1])$, to show that the $\operatorname{map} j:\left[Y, \mho_{1}\right]\left(=\Pi^{1}(Y)\right) \rightarrow\left[Y, \mathcal{Q}_{n}\right]$ given by

$$
j[f]=\left[\left(\begin{array}{cc}
f & 0 \\
0 & 1_{n-1}
\end{array}\right)\right],
$$

for $[f] \in\left[Y, \mathscr{Q}_{1}\right]$ is an isomorphism. The proof of the theorem is completed by observing that the following diagram is commutative:

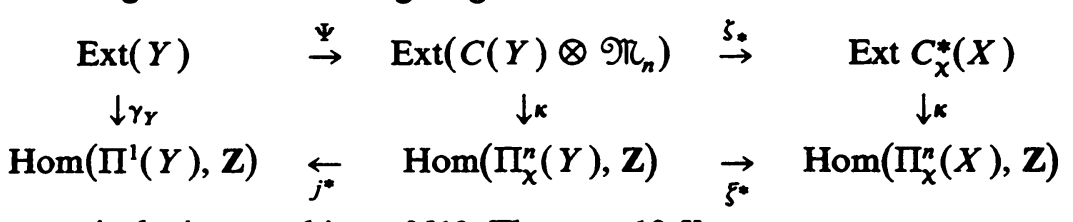

where $\gamma_{Y}$ is the isomorphism of [10, Theorem 10.5]. 
REMARK 3.13. Even when $X$ is a hypoconvex subset of $\mathcal{T} \mathscr{T} \zeta_{2}$, the map $\iota_{X}$ may not be injective. Indeed, let $Z$ be a compact subset of $C^{2}$ homeomorphic to the real projective plane, such that $\left|\lambda_{1}\right|>\left|\lambda_{2}\right|$ for every $\left(\lambda_{1}, \lambda_{2}\right) \in Z$. Let $Y \subset \Re_{2}$ defined by

$$
Y=\left\{\left(\begin{array}{cc}
\lambda_{1} & 1 \\
0 & \lambda_{2}
\end{array}\right):\left(\lambda_{1}, \lambda_{2}\right) \in Z\right\} .
$$

Then any two different matrices of $Y$ are inequivalent and irreducible and hence $Y$ is a generating set for $X=\hat{Y}$. From Theorem 3.9 and [19], it follows that $E N_{2}(X) \cong \operatorname{Ext}(Y) \cong Z_{2}$, and hence kernel $\iota_{X}=E N_{2}(X)=Z_{2} \neq\{0\}$ (notice that $\operatorname{Hom}\left(\Pi_{\chi}^{n}(X), Z\right)$ is torsion free).

Our next objective is to prove the homotopy invariance of $E N_{n}(\cdot)$ and $E N_{n}^{s}(\cdot)$. We start with the following lemma whose proof is elementary and will be omitted.

LEMMA 3.14. Let $X, Y \in \mathrm{HC}_{n} . \theta:[0,1] \rightarrow \operatorname{Hom}\left(C_{\chi}^{*}(Y), C_{\chi}^{*}(X)\right)$ is a continuous function, and if we define $h:[0,1] \rightarrow \operatorname{Hom}(X, Y)$ by $h(t)=[\theta(t)] x$, then $h$ is a continuous function, where $\operatorname{Hom}(X, Y)$ is endowed with the topology of uniform convergence (i.e. the topology inherited by that of $C_{X}^{*}(X)$ ). Conversely, if $h:[0,1] \rightarrow \operatorname{Hom}(X, Y)$ is a continuous function, then the function $\theta:[0,1] \rightarrow$ $\operatorname{Hom}\left(C_{\chi}^{*}(Y), C_{\chi}^{*}(X)\right)$ defined by $\theta(t)=h(t)^{*}, t \in[0,1]$, is also continuous.

Defintion 3.15. Given $f \in \operatorname{Hom}(X, Y)$ we denote by $f_{*}^{s}: E N_{n}^{s}(X) \rightarrow$ $E N_{n}^{s}(Y)$, and by $f_{*}: E N_{n}(X) \rightarrow E N_{n}(Y)$, the maps given by $f_{*}^{s}[T]_{s}=\left[S_{f}\right]_{s}$ and $f_{*}[T]=\left[S_{f}\right]$, where $S_{f}$ is any operator in $\mathcal{L}(\mathcal{S C})$ such that $\pi S_{f}=f(\pi T)$.

REMARK 3.16. It is easy to check that the maps $f_{*}^{s}$ and $f_{*}$ are well defined. On the other hand, since for each $f \in \operatorname{Hom}(X, Y), X, Y \in \mathbf{H C}_{n}$, the following diagrams commute:

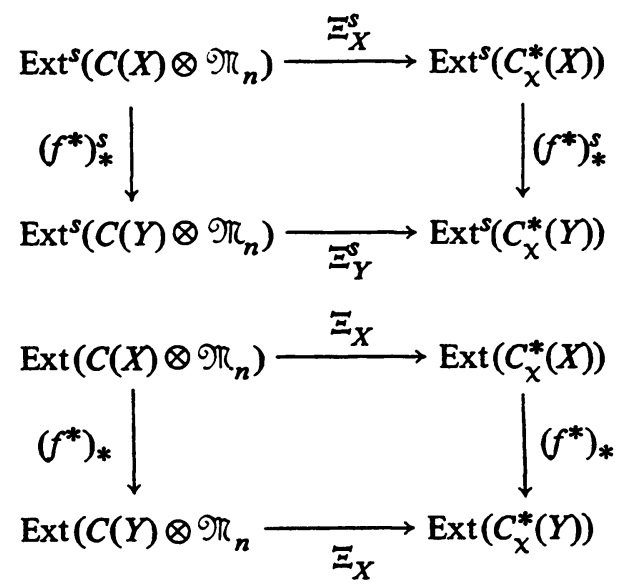


we deduce that the following diagrams are also commutative:
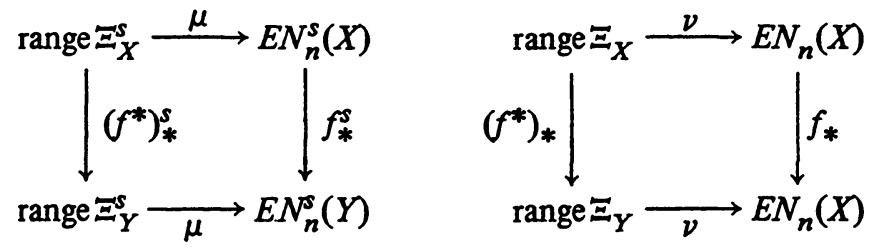

The above observations show that $E N_{n}^{s}(\cdot)$ and $E N_{n}(\cdot)$ have functorial properties. Indeed, we have the following theorem.

THEOREM 3.17. $E N_{n}^{s}(\cdot)$ and $E N_{n}(\cdot)$ are homotopy invariant covariant functors from the category $\mathbf{H C}_{n}$ into the category of abelian groups. In particular, if $X, Y \in \mathbf{H C}_{n}$, and $h:[0,1] \rightarrow \operatorname{Hom}(X, Y)$ is a continuous function, then $h(0)_{*}^{s}$ $=h(1)_{*}^{s}$, and $h(0)_{*}=h(1)_{*}$.

Proof. We only prove the theorem for the functor $E N_{n}^{s}(\cdot)$ since the proof for $E N_{n}(\cdot)$ is similar. For $X, Y, h$ as in the statement of the theorem and for $t \in[0,1]$, we see that the following diagrams are commutative:

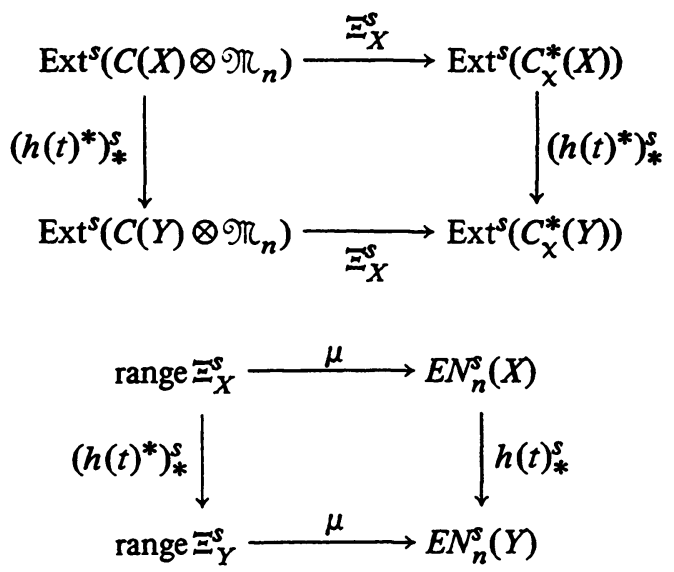

From Theorem 3.1 and Lemma 3.14 we conclude that $\left(h(0)^{*}\right)_{*}^{s}=\left(h(1)^{*}\right)_{*}^{s}$ and hence that $h(0)_{*}^{s}=h(1)_{*}^{s}$, as desired.

COROLlaRY 3.18. Let $\mathscr{G}_{n}$ be the unit ball in $\mathscr{T}_{n}$, then $E N_{n}^{s}\left(\mathscr{G}_{n}\right)=E N_{n}\left(\mathscr{B}_{n}\right)$ $=\{0\}$. In particular, if $T$ is an essentially n-normal operator in $\mathcal{E}(\mathcal{H C})$ such that $R_{e}^{n}(T)=\mathscr{B}_{n}$, then $T$ is a compact perturbation of n-normal operator $S$ in $\mathcal{E}(\mathcal{F C})$ such that $R_{e}^{n}(S)=\mathscr{B}_{n}$. 
Proof. Let $h:[0,1] \rightarrow \operatorname{Hom}\left(\mathscr{B}_{n}, \mathscr{B}_{n}\right)$ be given by $h(t)=t \chi$, where $t \in$ $[0,1]$ and $\chi$ is the identity function on $\mathscr{B}_{n}$. Then $h(1)_{*}^{s}$ is the identity on $E N_{n}^{s}\left(\mathscr{G}_{n}\right)$ while $h(0)_{*}^{s}$ is the zero homomorphism.

Our final task in this section is to present a partial answer to a question raised in [30].

REMARK 3.19. It is well known that if every irreducible representation of $C^{*}(T)$ is at most $n$-dimensional, then the operator $T$ can be expressed as $T=\sum_{k=1}^{n} \oplus T_{k}$, where $T_{k}$ is a $k$-normal and some summands might be missing (cf. [12]). In particular, if $C^{*}(T)$ is $n$-homogeneous, i.e. if every irreducible representation of $C^{*}(T)$ is $n$-dimensional, then $T$ must be $n$-normal. For $n>2$, it is not known (and likely to be false) whether the Calkin algebra version of the last fact holds. The following theorem shows that for $n<2$ this question has a positive answer.

THEOREM 3.20. If every irreducible representation $\pi\left(C^{*}(T)\right)$ is 2-dimensional, then the operator $T$ is essentially binormal.

ProOf. It readily follows that if every irreducible representation of $\pi\left(C^{*}(T)\right)$ is at most 2-dimensional, then the map $\Gamma_{\pi T}: C^{*}(\pi T) \rightarrow C_{\chi}^{*}\left(R^{2}(\pi T)\right)$ (cf. Definition 2.1) is an isomorphism. Now let $\tau=\left(\Gamma_{\pi T}\right)^{-1}$, and let $X=$ $R^{2}(\pi T)\left(=R_{e}^{2}(T)\right)$. Since, by hypothesis, every irreducible representation of $\pi\left(C^{*}(T)\right.$ ) (and hence of $C_{x}^{*}(X)$ ) is 2 dimensional, we deduce that $X$ is a hypoconvex subset of $G \mathbb{R}_{2}$. From Remark 2.10 we then see that there exists a generating set for $X$. As we remarked previously, the inclusion map $Y \rightarrow X$ induces a $*$-isomorphism $\zeta: C_{\chi}^{*}(X) \rightarrow C_{\chi}^{*}(Y)$ and a *-homomorphism $\theta$ : $C(X) \otimes \mathbb{K}_{2} \rightarrow C(Y) \otimes \mathbb{K}_{2}$. Therefore, the following diagram is commutative:

$$
\begin{array}{ccc}
C(Y) \times \Re_{2} & \stackrel{\theta_{*}}{\rightarrow} & C(X) \times \Re_{2} \\
\text { (id) } \downarrow & & \downarrow \Xi_{X} \\
C_{x}^{*}(Y) & \overrightarrow{\zeta^{*}} & C_{\chi}^{*}(X)
\end{array}
$$

Since (id) $)_{*}$ and $\zeta_{*}$ are isomorphisms, it follows that $\Xi_{X}$ is surjective. In particular, if $\tau$ is as above, then $[\tau] \in$ range of $\Xi_{X}$, and hence $T$ is essentially binormal.

4. Toeplitz operators on strongly pseudoconvex domains. In this section we apply the results of preceding ones to obtain a homotopic property of Toeplitz operators with matricial continuous symbols on strongly pseudoconvex domains. We start by proving some few facts concerning the $C^{*}$-algebra generated by the set of Toeplitz operators. Our presentation follows the pattern of [15]. 
In what follows, $\Omega$ will denote a strongly pseudoconvex domain (also called strictly Levi-pseudoconvex, cf. [20]) in $\mathbf{C}^{m}$ with smooth boundary. $L^{2}(\Omega)$ will denote the Hilbert space of all complex valued square integrable functions on $\Omega$ with respect to Lebesgue measure, and $H^{2}(\Omega)$ will denote the subspace of all holomorphic functions in $L^{2}(\Omega)$. We shall denote by $P: L^{2}(\Omega) \rightarrow H^{2}(\Omega)$ the (orthogonal) projection onto $H^{2}(\Omega)$, and $k(z, w),(z, w) \in \Omega \times \Omega$ (the Bergman kernel [5]) will denote the reproducing kernel of $H^{2}(\Omega)$. We recall that for each $w \in \Omega, k(\cdot, w) \in H^{2}(\Omega)$ and for every $f \in L^{2}(\Omega), \quad P f(z)=$ $\int_{\Omega} k(z, w) f(w) d w$. In particular, $k(z, w)=\sum_{j=1}^{\infty} g_{j}(z) \bar{g}_{j}(w)$, where $\left\{g_{j}, j=\right.$ $1,2, \ldots\}$ is an orthonormal basis for $H^{2}(\Omega)$. From [23] it follows that $k(z, w)$ is a $C^{\infty}$ function up to the boundary of $\Omega \times \Omega$, except for the diagonal $\{(z, w) \in \partial \Omega \times \partial \Omega: z=w\}$. Also, for $z \in \Omega, k(z, z)>0$, and $\lim _{z \rightarrow z_{0}} k(z, z)$ $=\infty$ for every $z_{0} \in \partial \Omega$ (see [7]).

Defintion 4.1 (SEE [33]). Given $\varphi \in C(\bar{\Omega})$, we denote by $M_{\varphi}$ the operator on $L^{2}(\Omega)$ defined by multiplication by $\varphi$, and by $T_{\varphi}$ the operator on $H^{2}(\Omega)$ defined by $T_{\varphi} f=P \varphi f=P M_{\varphi} f, f \in H^{2}(\Omega)$. Also, the norm closed subalgebra of $\mathcal{L}\left(L^{2}(\Omega)\right)$ generated by all the operators $T_{\varphi}, \varphi \in C(\bar{\Omega})$, will be denoted by T( $(\Omega)$.

LEMMA 4.2. Let $\varphi \in C(\bar{\Omega})$.

(a) $P M_{\varphi}-M_{\varphi} P$ is compact on $L^{2}(\Omega)$.

(b) $T_{\varphi}$ is compact on $H^{2}(\Omega)$ if and only if $\varphi \mid \partial \Omega=0$.

Proof. Statement (a) was proved in [33, Theorem 2.1] for the case that $\varphi \in C^{\infty}(\Omega)$. Since these functions are dense in $C(\bar{\Omega})$ and $\mathcal{K}\left(L^{2}(\Omega)\right)$ is closed, the validity of part (a) follows. Furthermore, in [33, Theorem 2.3] it was shown that if $\varphi \mid \partial \Omega=0$ and $\varphi \in C^{\infty}(\Omega)$, then $T_{\varphi}$ is compact. Thus one half of part (b) follows using the same argument employed previously. For the other half of part (b), suppose that $T_{\varphi}$ is compact for some $\varphi \in C(\bar{\Omega})$. Let $z_{0}$ be an arbitrary point in $\partial \Omega$, let $\left\{z_{j}\right\}$ be a sequence in $\Omega$ that converges to $z_{0}$, and let $f_{j}$ be the function in $H^{2}(\Omega)$ defined by $f_{j}(z)=k\left(z, z_{j}\right) /\left[k\left(z_{j}, z_{j}\right)\right]^{1 / 2}, j=$ $1,2, \ldots, z \in \Omega$. It readily follows, using the fact that $k$ is a reproducing kernel, that $\left\|f_{j}\right\|=1$ for $j=1,2, \ldots$ Also, from [23] we see that for every open neighborhood $\omega$ of $z_{0}, f_{j} \in C^{\infty}(\bar{\Omega} \backslash \omega)$ and converges uniformly to zero on $\bar{\Omega} \backslash \omega$. Therefore,

$$
\lim _{j \rightarrow \infty}\left\|\left[T_{\varphi}-\varphi\left(z_{0}\right)\right] f_{j}\right\|^{2} \leqslant \lim _{j \rightarrow \infty} \int_{\Omega}\left|\varphi(w)-\varphi\left(z_{0}\right)\right|^{2}\left|f_{j}(w)\right|^{2} d w=0 .
$$

On the other hand, using again the fact that $k$ is a reproducing kernel, we see that for every bounded $f \in H^{2}(\Omega)$,

$$
\lim _{j \rightarrow \infty}\left\langle f, f_{j}\right\rangle=\lim _{j \rightarrow \infty} f\left(z_{j}\right) /\left[k\left(z_{j}, z_{j}\right)\right]^{1 / 2}=0
$$

and hence $\lim _{j \rightarrow \infty}\left\langle f, f_{j}\right\rangle=0$ for every $f \in H^{2}(\Omega)$. Here we have used the 
standard fact that $H^{\infty}(\Omega)$ is dense in $H^{2}(\Omega)$. Thus, $\left\{f_{j}\right\}$ converges weakly to zero, and since $T_{\Phi}$ is compact, we see that $\lim _{j \rightarrow \infty}\left\|T_{\Phi} f_{j}\right\|=0$. We conclude that

$$
\left|\varphi\left(z_{0}\right)\right|<\lim _{j \rightarrow \infty}\left\{\left\|\left[T_{\varphi}-\varphi\left(z_{0}\right)\right] f_{j}\right\|+\left\|T_{\varphi} f_{j}\right\|\right\}=0
$$

as desired.

REMARK 4.3. Reasoning just as in [15, Lemma 1] it follows that $\mathscr{T}(\Omega)$ is an irreducible $C^{*}$-algebra on $H^{2}(\Omega)$, and since it clearly contains nonzero compact operators, we see that $\mathcal{K}\left[H^{2}(\Omega)\right] \subset \mathcal{T}(\Omega)$. Let $\varphi \in C(\partial \Omega)$ and let $\varphi^{0} \in$ $C(\bar{\Omega})$ be any continuous extension of $\varphi$. It follows from Lemma 4.2 that the element $\pi T_{\varphi^{0}}$ in $\mathcal{2}\left[H^{2}(\Omega)\right]$ is independent of the choice of the extension $\varphi^{0}$ of $\varphi$.

LEMMA 4.4. Let $\tau: C(\partial \Omega) \rightarrow \mathcal{2}\left[H^{2}(\Omega)\right]$ be defined by $\tau \varphi=\pi T_{\varphi^{0}}, \varphi \in C(\partial \Omega)$. Then $\tau \in E[C(\partial \Omega)]$ (i.e., $\tau$ is a unital *-monomorphism).

Proof. In view of Lemma 4.2(b) we need only check that $\tau$ is a homomorphism. Let $\varphi, \psi \in C(\partial \Omega)$. Then,

$$
\begin{aligned}
(\tau \varphi)(\tau \psi) & =\left(\pi T_{\varphi^{0}}\right)\left(\pi T_{\psi^{0}}\right)=\pi T_{\varphi^{0}} T_{\psi^{0}} \\
& =\pi\left(T_{\varphi^{\circ} \psi^{0}}-P M_{\varphi^{0}}(1-P) M_{\psi^{0}} \mid H^{2}(\Omega)\right)=\pi T_{\varphi^{\circ} \psi^{0}}=\tau(\varphi \psi),
\end{aligned}
$$

where we have used Lemma 4.2(a).

Defintion 4.5. Let $\mathcal{X}=H^{2}(\Omega) \otimes C^{n}$ and let $P_{n}=P \otimes 1_{n}$, where $1_{n}$ is the identity matrix in $\mathscr{T}_{n}$. Given $\varphi \in C(\Omega) \otimes \Re_{n}$ define $T_{\varphi}$ on $\mathcal{T}$ by $T_{\varphi} x=$ $P_{n} \varphi x, x \in \mathcal{H}$. Also, let $\mathcal{T}_{n}(\Omega)$ be the norm closed subalgebra of $\mathcal{L}(\mathcal{H C})$ generated by $T_{\varphi}$, for $\varphi \in C(\partial \Omega) \otimes \Re_{n}$.

REMARK 4.6. An easy verification shows that $\mathcal{T}_{n}(\Omega)$ can be identified with $T(\Omega) \otimes \Re_{n}$. On the other hand, from Lemma 4.4, it follows that the sequence

$$
0 \rightarrow \mathscr{K}\left[H^{2}(\Omega)\right] \rightarrow \sigma(\Omega) \rightarrow C(\partial \Omega) \rightarrow 0
$$

is exact. Since tensoring by $\mathscr{T}_{n}$ preserves exactness and $\mathscr{K}_{\left[H^{2}(\Omega)\right] \otimes \mathfrak{K}_{n}}$ can be naturally identified with $\mathcal{K}(\mathcal{H})$, we deduce that the following sequence is also exact

$$
0 \rightarrow \mathscr{K}(\mathcal{H}) \rightarrow \tau_{n}(\Omega) \rightarrow C(\partial \Omega) \otimes \Re_{n} \rightarrow 0 .
$$

As in Remark 4.3, given $\varphi \in C(\partial \Omega) \otimes \mathscr{T}_{n}$ we denote by $\varphi^{0}$ any continuous extension of $\varphi$ to $\bar{\Omega}$.

The following corollary is a consequence of Lemma 4.4 and the above remark.

COROLLARY 4.7. Let $\tau_{n}: C(\partial \Omega) \otimes \pi_{n} \rightarrow \mathcal{2}(\mathcal{H C})$ given by $\tau_{n} \varphi=\pi T_{\varphi^{0}}, \varphi \in$ $C(\partial \Omega) \otimes \mathfrak{T}_{n}$. Then $\tau_{n} \in E\left[C(\partial \Omega) \otimes \mathfrak{R}_{n}\right]$. In fact, $\tau_{n}=\tau \otimes 1_{n}$, where $\tau$ is as in Lemma 4.4. 
With the above result we are finished with the preliminary material needed for the application of the previous sections.

THEOREM 4.8. Let $\varphi, \psi \in C(\partial \Omega) \otimes \Re_{n}$ and suppose that $X \in \mathbf{H C}_{n}$ and that range $\varphi \subset X$, range $\psi \subset X$. Furthermore, assume that there exist a continuous function $h:[0,1] \rightarrow C(\partial \Omega) \otimes \Re_{n}, h(0)=\varphi, h(1)=\psi$, and range $h(t) \subset X$ for every $0<t<1$. Then, for every n-normal operator $S$ in $\mathcal{L}(\mathcal{H})$ such that $R_{e}^{n}(S)=X$, we have $T_{\varphi^{0}} \oplus S \sim_{s} T_{\psi^{0}} \oplus S$. Moreover, if [range $\left.\varphi\right]^{\wedge}=$ [range $\psi$ ] $=X$, it follows that $T_{\varphi^{0}} \sim_{s} T_{\psi^{0}}$, i.e. there exists a unitary $U$ in $\mathcal{E}(\mathcal{F C})$ such that $U^{*} T_{\varphi^{\circ}} U-T_{\psi^{0}} \in \mathcal{K}(\mathcal{H})$.

Proof. Let $\varphi^{*}: C_{\chi}^{*}(X) \rightarrow C(\partial \Omega) \otimes \Re_{n}$ defined by $\varphi^{*} f=f \circ \varphi, f \in C_{\chi}^{*}(X)$. Furthermore, let $\theta:[0,1] \rightarrow \operatorname{Hom}\left(C_{x}^{*}(X), C(\partial \Omega) \otimes \Re_{n}\right)$ be defined by $\theta(t)=$ $h(t)^{*}, 0<t<1$. Then it readily follows that $\theta$ is a continuous function, $\theta(0)=\varphi^{*}$ and $\theta(1)=\psi^{*}$. Since every irreducible representation of $C_{\chi}^{*}(X)$ and $C(\partial \Omega) \otimes \Re_{n}$ is at most $n$-dimensional (see [17, Chapter 3]), from Theorem 3.1 we deduce that $\theta(0)_{*}^{s}=\theta(1)_{*}^{s}$. In particular, $\left(\varphi^{*}\right)_{*}^{s}\left[\tau_{n}\right]_{s}=$ $\left(\psi^{*}\right)_{*}^{s}\left[\tau_{n}\right]_{s}$. This means that for $\sigma \in E_{0}\left(C(X) \otimes \Re_{n}\right)$, there exists a unitary $U$ in $E(\mathcal{E C})$ such that

$$
\pi U^{*}\left[\tau_{n}(f \circ \varphi) \oplus \sigma f\right] \pi U=\tau_{n}(f \circ \psi) \oplus \sigma f, \quad \text { for every } f \in C_{X}^{*}(X) .
$$

Since $\tau_{n} \in E\left[C(\partial \Omega) \otimes \Re_{n}\right]$, the restriction of $\tau_{n}$ to $C^{*}(\varphi)$ defines a *-isomorphism from $C^{*}(\varphi)$ onto $C^{*}\left(\pi T_{\varphi^{0}}\right)$. From Corollary 2.4 it follows that $R_{e}^{n}\left(T_{\varphi^{0}}\right)$ $=[\text { range } \psi]^{\wedge} \subset X$. Let $S$ in $\mathcal{L}(\mathcal{H})$ be an $n$-normal operator such that $\pi S=\sigma \chi$ (see Corollary 3.6). Then, it follows that $R_{e}^{n}(S)=X$ and, applying (\#) for $f=\chi$, we obtain: $U^{*}\left(T_{\varphi^{\circ}} \oplus S\right) U-T_{\psi^{\circ}} \oplus S \in \mathcal{K}(\mathcal{H C})$. Now, if [range $\left.\varphi\right]^{0}=$ [range $\psi]^{0}=X$, then, as noted previously, $R_{e}^{n}\left(T_{\varphi^{0}}\right)=R_{e}^{n}\left(T_{\psi^{0}}\right)=X$. Therefore, from the results mentioned in Remark 1.5 (see [27]) we conclude that $T_{\varphi^{0}} \sim_{s} T_{\varphi^{0}} \oplus S \sim_{s} T_{\psi^{0},}$ as required.

REMARK 4.9. (a) The converse of the last part of Theorem 4.8 is false in general (at least in lower dimensions). What is too strong is the homotopy condition, because the condition about the ranges of $\varphi$ and $\psi$ is a necessary one. Let $X$ be a "figure 8 ", i.e.

$$
X=\{\lambda \in \mathbf{C}:|\lambda-1|=1\} \cup\{\lambda \in \mathbf{C}:|\lambda+1|=1\} .
$$

Let $\varphi$ and $\psi$ be the complex valued continuous functions on the unit circle $S^{1}$ defined by

$$
\begin{aligned}
& \varphi(\lambda)= \begin{cases}1+\lambda^{2}, & |\arg \lambda|<\pi / 2, \\
-1-\lambda^{2}, & \pi / 2<|\arg \lambda|<\pi ;\end{cases} \\
& \psi(\lambda)= \begin{cases}-1-\lambda^{2}, & |\arg \lambda|<\pi / 2, \\
1+\lambda^{2}, & \pi / 2<|\arg \lambda|<\pi .\end{cases}
\end{aligned}
$$


Then, if $\omega(f)$ denotes the winding number of an invertible function $f \in$ $C\left(S^{1}\right)$ about the origin, we have $\omega(\varphi-1)=\omega(\psi-1)=\omega(\varphi+1)=\omega(\psi+$ $1)=1$. Since range $\varphi=$ range $\psi=X$, and $T_{\varphi}$ and $T_{\psi}$ are essentially normal operators, it follows that the essential spectra of $T_{\varphi}$ and $T_{\psi}$ coincide with $X$, and ind $\left(T_{\varphi}-\lambda\right)=\operatorname{ind}\left(T_{\psi}-\lambda\right)$, for every $\lambda \notin X$. Therefore, from [10, Theorem 11.1] $T_{\varphi} \sim_{s} T_{\psi}$. However, $\varphi$ and $\psi$ are not in the same homotopy class of the fundamental group $\pi_{1}(X)$, because this is a free group on two generators, and $[\varphi]$ and $[\psi]$ represent two different reduced words. It would be interesting to find out whether the phenomenon presented here is due to the noncommutativity of the fundamental group of $X$.

(b) Let $\varphi$ and $\psi \in C\left(S^{1}\right) \otimes T_{2}$ given by $\varphi(\lambda)=\left(\begin{array}{ll}\lambda & 1 \\ 0 & \lambda\end{array}\right), \psi(\lambda)=\left(\begin{array}{ll}\lambda & 2 \\ 0 & \lambda\end{array}\right), \lambda \in S^{1}$. $T_{\varphi^{0}}$ and $T_{\psi^{0}}$ are compact perturbations of $\left(\begin{array}{ll}U^{+} & 1 \\ 0 & U^{+}\end{array}\right)$and $\left(\begin{array}{ll}U^{+} & 2 \\ 0 & U^{+}\end{array}\right)$, respectively, where $U^{+}$is a simple unilateral shift. Let $Y \subset T_{2}$ be the set of all matrices of the form $\left.Y=\left\{\begin{array}{cc}\lambda & \alpha \\ 0 & \lambda\end{array}\right),|\lambda|=1,1<\alpha<2\right\}$. Then [range $\left.\varphi\right]^{\wedge} \subset \hat{Y}$ and [range $\psi$ ] $\subset \hat{Y}$. If $S$ is any binormal operator such that $R_{e}^{2}(S)=\hat{Y}$ (which can be obtained by using Corollary 1.12), then, from Theorem 4.8, $\left(\begin{array}{ll}U_{0}^{+} & 1 \\ U^{+}\end{array}\right) \oplus$ $S$ is strongly equivalent to $\left(0_{0}^{U^{+}} U^{+}\right) \oplus S$. The idea of this example was suggested to us by Vern Paulsen, who proved that $\left(U_{0}^{U^{+}}{ }_{U^{+}}^{2}\right) \oplus S$ is weakly equivalent to $\left(\begin{array}{ll}U_{0}^{+} & 2 \\ U^{+}\end{array}\right) \oplus S$ employing the invariant that he introduced in [25] and the classification of essentially normal operators discovered by Brown, Douglas and Fillmore [10, Theorem 11.1].

Defintion 4.10. Let $X$ be a hypoconvex subset of $\Re_{n}$ and let $L \in X$. Let $\xi_{m}: \Pi_{m}(X, L) \rightarrow E N_{n}(X)$ be the map given by $\xi_{m}[\varphi]=\left[T_{\varphi^{\circ}} \oplus S\right]$, where $S$ is some $n$-normal operator in $\mathcal{E}(\mathcal{H C})$ such that $R_{e}^{n}(S)=X$. Hereafter, $\Pi_{m}(X, L)$ denotes the $(2 m-1)$ th homotopy group of $X$ with base point $L$ [32, Chapter 7 , §2]. In the following theorem we see that $\xi_{m}$ resembles the Hurewicz homomorphism [32, Chapter 7, §4].

THEOREM 4.11. Let $B_{m}$ be the closed unit ball in $\mathbf{C}^{m}$. If $\tau_{n} \in E\left[C\left(\partial B_{m}\right) \otimes\right.$ $\Re_{n}$ ], and $\nu$ : range $\Xi_{X} \rightarrow E N_{n}(X)$ are defined as in Corollary 4.7 and Remark 3.7 , then $\xi_{m}[\varphi]=\left(\nu \circ\left(\varphi^{*}\right)_{*}\right)\left[\tau_{n}\right]$, for every $[\varphi] \in \Pi_{m}(X, L)$. Furthermore, $\xi_{m}$ is a group homomorphism.

Proof. Since the first part of the theorem follows immediately from the definitions of the maps involved, we shall be concerned with the second half. Let $Z_{1}=\partial B_{m}=Z_{2}$, let $Z$ be the disjoint union of $Z_{1}$ and $Z_{2}$ with the base point identified, and let $g: \partial B_{m} \rightarrow Z$ be the map that collapses the equator of $\partial B_{m}$ to the base point. Let $\left[\varphi_{j}\right] \in \Pi_{m}(X, L)$, define $\varphi: Z \rightarrow X$ such that $\varphi \mid Z_{j}=\varphi_{j}, j=1,2$. Then the product of $\left[\varphi_{1}\right]$ and $\left[\varphi_{2}\right]$ in $\Pi_{m}(X, L)$ is defined as $\left[\varphi_{1}\right] \circ\left[\varphi_{2}\right]=[\varphi \circ g]$ (recall that this operation is abelian for $m>1$, cf. [32, Chapter 7, §2]). Let $i_{j}: Z_{j} \rightarrow Z$ be the canonical inclusion maps and let $\beta(a, b)=i_{1 *} a+i_{2 *} b, a \in \operatorname{Ext}\left(Z_{1}\right), b \in \operatorname{Ext}\left(Z_{2}\right)$. From [10, Corollary 6.2] it follows that $\beta$ is an isomorphism. A right inverse for $\beta$ is easily seen to be the 
map $\left(r_{1 *}, r_{2 *}\right)$ where $r_{j}: Z \rightarrow Z_{j}$ is the retraction obtained by collapsing $Z_{3-j}$ to the base point, $j=1,2$. Now notice that $r_{j} g: \partial B_{m} \rightarrow \partial B_{m}$ is homotopic to the identity map, so that $\left(\left(r_{1 *}, r_{2 *}\right) \circ g_{*}\right)\left[\tau_{n}\right]=\left(\left(r_{1} \circ g\right)_{*},\left(r_{2} \circ g\right)_{*}\right)\left[\tau_{n}\right]=\left(\left[\tau_{n}\right]\right.$, $\left.\left[\tau_{n}\right]\right)$. On the other hand,

$$
\begin{aligned}
\left(\varphi^{*}\right)_{*} \circ \beta & =\left(\varphi^{*}\right)_{*} \circ\left(i_{1 *}+i_{2 *}\right) \\
& =\left(\left(\varphi \circ i_{1}\right)^{*}\right)_{*}+\left(\left(\varphi \circ i_{2}\right)^{*}\right)_{*}=\left(\varphi_{1}^{*}\right)_{*}+\left(\varphi_{2}^{*}\right)_{*}
\end{aligned}
$$

Therefore,

$$
\begin{aligned}
\xi_{m}\left(\left[\varphi_{1}\right] \circ\left[\varphi_{2}\right]\right) & =\left(\nu \circ\left((\varphi \circ g)^{*}\right)_{*}\right)\left[\tau_{n}\right]=\left(\nu \circ\left(\varphi^{*}\right)_{*} \circ g_{*}\right)\left[\tau_{n}\right] \\
& =\left(\nu \circ\left(\varphi^{*}\right)_{*} \beta\left(r_{1 *}, r_{2 *}\right) \circ g_{*}\right)\left[\tau_{n}\right] \\
& =\left(\nu \circ\left(\varphi_{1}^{*}\right)_{*}\right)\left[\tau_{n}\right]+\left(\nu \circ\left(\varphi_{2}^{*}\right)_{*}\right)\left[\tau_{n}\right] \\
& =\xi_{m}\left[\varphi_{1}\right]+\xi_{m}\left[\varphi_{2}\right] .
\end{aligned}
$$

The proof of the theorem is complete.

REMARK 4.12. From Corollary 4.7 it follows that if $\varphi$ is invertible in $C(\partial \Omega) \otimes \Re_{n}$, then $T_{\varphi^{\circ}}$ is a Fredholm operator in $\mathcal{L}(\mathcal{H})$. In [33] it was shown that if $\Omega=B_{m}$, and $m \leqslant n$, then ind $T_{\varphi^{\circ}}=(-1 /(n-1) !) \operatorname{deg} \tilde{\varphi}$, where deg $\tilde{\varphi}$ denotes the degree of $\tilde{\varphi}$ and $\tilde{\varphi}: \partial B_{m} \rightarrow \partial B_{m}$ is obtained by deforming first the continuous $n \times n$ matrix valued function $\varphi$ to one of the form $\left(\begin{array}{l}\varphi^{\prime} \\ 0 \\ 1_{n-m}\end{array}\right)$, where $\varphi^{\prime} \in C\left(\partial B_{m}\right) \otimes \Re_{m}$, and then defining $\tilde{\varphi}(z)=\varphi_{1}^{\prime}(z) /\left|\varphi_{m}^{\prime}(z)\right|$, where $\varphi_{1}^{\prime}(z)$ is the first column of the matrix $\varphi^{\prime}(z), z \in \partial B_{m}$. This deformation can be performed by using the standard argument with the homotopy sequence of the fibration $\mathcal{Q}_{k} \rightarrow \mathcal{Q}_{k+1} \rightarrow \partial B_{k+1}, k=m, m+1, \ldots$, as mentioned in the proof of Theorem 3.17 (see also [4, p. 239]).

DefINITION 4.13. For $m \leqslant n, X \in \mathbf{H C}_{n}$, let $\delta_{m}: \Pi_{m}(X, L) \rightarrow$ $\operatorname{Hom}\left(\Pi_{\chi}^{n}(X), Z\right)$ be the homomorphism given by

$$
\delta_{m}[\varphi]([f])=(-1 /(m-1) !) \operatorname{deg} \widetilde{(f \circ \varphi)} .
$$

THEOREM 4.14. Let $m<n, X \in \mathrm{HC}_{n}$; then the following diagram is commutative:

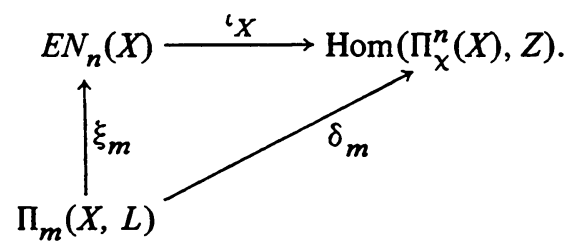

ProOF. By using the fact that an $n$-normal operator is quasidiagonal (see for instance [27, Theorem 3.4]), it is easy to see that if $S$ is a Fredholm $n$-normal operator in $\mathcal{L}(\mathcal{H})$, then its index is zero. Now let $[\varphi] \in \Pi_{m}(X, L)$ and let $[f] \in \Pi_{\chi}^{n}(X)$. Then, employing [33, Theorem 1.5] we deduce that for 
every $[\varphi] \in \Pi_{m}(X, L),[f] \in \Pi_{\chi}^{n}(X)$, we have

$$
\begin{aligned}
\left(\left(\iota_{X} \circ \xi_{m}\right)[\varphi]\right)[f] & =\left(\iota_{X} \nu\left(\varphi^{*}\right)_{*}\left[\tau_{n}\right]\right)[f]=\operatorname{ind}\left(\left(\pi T_{\varphi^{\circ} \circ f}\right) \oplus \pi \sigma f\right) \\
& =\operatorname{ind}\left(T_{\varphi^{\circ} \circ f}\right)=(-1 /(m-1) !) \operatorname{deg} \widehat{(\varphi \circ f)}=\left(\delta_{m}[\varphi]\right)[f],
\end{aligned}
$$

where $\sigma \in \operatorname{Hom}\left(C_{x}^{*}(X), \mathcal{L}(\mathcal{H})\right)$ and $\left[\sigma C_{x}^{*}(X)\right] \cap \mathcal{K}(\mathcal{H})=\{0\}$. The proof of the theorem is complete.

REMARK 4.15. As pointed out already in Remark 4.9(a), the map $\xi_{1}$ is not always injective. On the other hand, from Theorem 4.14 it can be deduced that $\xi_{1}$ is not always surjective. Indeed, this is the case when $X$ is the subset of $\mathbf{R}^{2}$ which is the closure of

$$
\{(x,|\sin (\pi / x)|): 0<x<1\} \cup\{(x,-\sin \pi x), 0<x<1\} .
$$

It is reasonable to expect that, for $n>1$ and some suitable notion for higher order connectivity of a hypoconvex subset $X$ in $\Re_{n}$, the map $\xi_{n}$ be an isomorphism. More generally, we believe, that at least in the case in which $X$ is arcwise connected and $E N_{n}(X)=E N_{n}^{s}(X)$, the knowledge of $\Pi_{m}(X)$, $1<m<n$, will provide enough information about the hypoconvex set $X$ in $\mathscr{T}_{n}$ so that $E N_{n}(X)$ can be effectively computed for $n>1$. To this end, the problems to be considered are the following:

(a) Is $\cap_{1<m<n} \operatorname{ker} \xi_{m}=\{0\}$ ?

(b) Given any $[T] \in E N_{n}(X)$, does there exist a function $\varphi \in$ $C\left(\partial B_{m}\right) \otimes \Re_{n}$, for $1<m<n$, and an $n$-normal operator $S$ in $\mathcal{L}(\mathcal{H C})$ with $R_{e}^{n}(S)=X$, such that $T \sim T_{\varphi^{0}} \oplus S$ ?

\section{REFERENCES}

1. T. B. Andersen, Linear extensions, projections and split faces, J. Functional Analysis 17 (1974), 161-173.

2. W. Arveson, A note on essentially normal operators, Proc. Roy. Irish Acad. 74 (1974), 143-146.

3. $\longrightarrow$ Notes on extensions of $C^{*}$-algebras, Duke Math. J. 44 (1977), 329-355.

4. M. F. Atiyah, Algebraic topology and elliptic operators, Comm. Pure Appl. Math. 20 (1967), 237-249.

5. S. Bergman, The kernel function and conformal mapping, rev. ed., Math. Surveys, no. 5, Amer. Math. Soc., Providence, R. I., 1970.

6. R. Bott, The periodicity theorem for the classical groups and its applications, Advances in Math. 4 (1970), 353-411.

7. L. Boutet de Monvel et J. Sjöstrand, Sur la singularité des noyaux de Bergman et de Szegö, Preprint.

8. A. Brown, The unitary equivalence of binormal operators, Amer. J. Math. 76 (1954), 414-434.

9. L. G. Brown, Extensions and the structure of $C^{*}$-algebras, Symposia Mathematica, vol. 20, Academic Press, New York, 1976, pp. 539-566.

10. L. G. Brown, R. G. Douglas and P. A. Fillmore, Unitary equivalence modulo the compact operators and the extensions of $C^{*}$-algebras, Proc. Conf. on Operator Theory, Lecture Notes in Math., vol. 345, Springer-Verlag, New York, 1973, pp. 58-128.

11. , Extensions of $C^{*}$-algebras and K-homology, Ann. of Math. (2) 105 (1977), 265-324. 
12. J. Bunce and J. Deddens, Irreducible representations of the C*-algebra generated by n-normal operators, Trans. Amer. Math. Soc. 171 (1972), 301-307.

13. J. Bunce and N. Salinas, Completely positive maps on $C^{*}$-algebras and the left matricial spectra of an operator, Duke Math. J. 43 (1976), 747-774.

14. J. Calkin, Two-sided ideals and congruence in the ring of bounded operators in Hilbert space, Ann. of Math. (2) 42 (1941), 839-973.

15. L. A. Coburn, Singular integral operators and Toeplitz operators on odd spheres, Indiana Univ. Math. J. 23 (1973), 433-439.

16. M. D. Choi and E. G. Effros, The completely positive lifting problem, Ann. of Math. (2) 104 (1976), 585-609.

17. J. Dixmier, Les $C^{*}$-algèbres et leurs représentations, Gauthier-Villars, Paris, 1969.

18. R. G. Douglas, Banach algebra techniques in operator theory, Academic Press, New York, 1972.

19. ,The relation of Ext to $K$-theory, Symposia Mathematica, vol. 20, Academic Press, New York, 1976, pp. 513-531.

20. R. Gunning and H. Rossi, Analytic functions of several complex variables, Prentice-Hall, Englewood Cliffs, N. J., 1965.

21. L. Hormander, $L^{2}$ estimates and existence theorems for the $\delta$ operator, Acta Math. 113 (1965), 89-152.

22. J. Kaminker and C. Schochet, Topological obstructions to perturbations of pairs of operators, $K$-Theory and Operator Algebras, Lecture Notes in Math., vol. 575, Springer-Verlag, Berlin, 1977, pp. 70-75.

23. N. Kerzman, The Bergman kernel function. Differentiability at the boundary, Math. Ann. 195 (1972), 149-158.

24. V. Paulsen, Continuous canonical forms for matrices under unitary equivalence, Pacific $J$. Math. 76 (1978).

25. , Weak compalence invariants for essentially n-normal operators, Amer. J. Math. (to appear).

26. C. Pearcy and N. Salinas, Finite dimensional representations of $C^{*}$-algebras and the reducing matricial spectra of an operator, Rev. Roumaine Math. Pures Appl. 20 (1975), 567-598.

27. $423-434$. , The reducing essential matricial spectra of an operator, Duke Math. J. 42 (1975),

28. , Extensions of $C^{*}$-algebras and the reducing essential matricial spectra of an operator, $K$-Theory and Operator Algebras, Lecture Notes in Math., vol. 575, Springer-Verlag, Berlin, 1977, pp. 96-112.

29. N. Salinas, Reducing essential eigenoalues, Duke Math. J. 40 (1973), 561-580.

30. Soc. 82 (1976), 143-146.

31. __ Homotopy invariance of $\operatorname{Ext}(\&)$, Duke Math. J. 44 (1977), 777-794.

32. E. H. Spanier, Algebraic topology, McGraw-Hill, New York, 1966.

33. V. Venugopalkrishna, Fredholm operators associated with strongly pseudoconvex domains in $C^{n}$, J. Functional Analysis 9 (1972), 349-373.

34. D. Voiculescu, A non-commutative Weyl-von Neumann theorem, Rev. Roumaine Math. Pures Appl. 21 (1976), 97-113.

35. H. Weyl, Über beschrankte quadratischen Formen deren Differenz vollstetig ist, Rend. Circ. Mat. Palermo 27 (1909), 373-392.

Department of Mathematics, Universtry OF KANSAS, LAWREnCE, Kansas 66045 